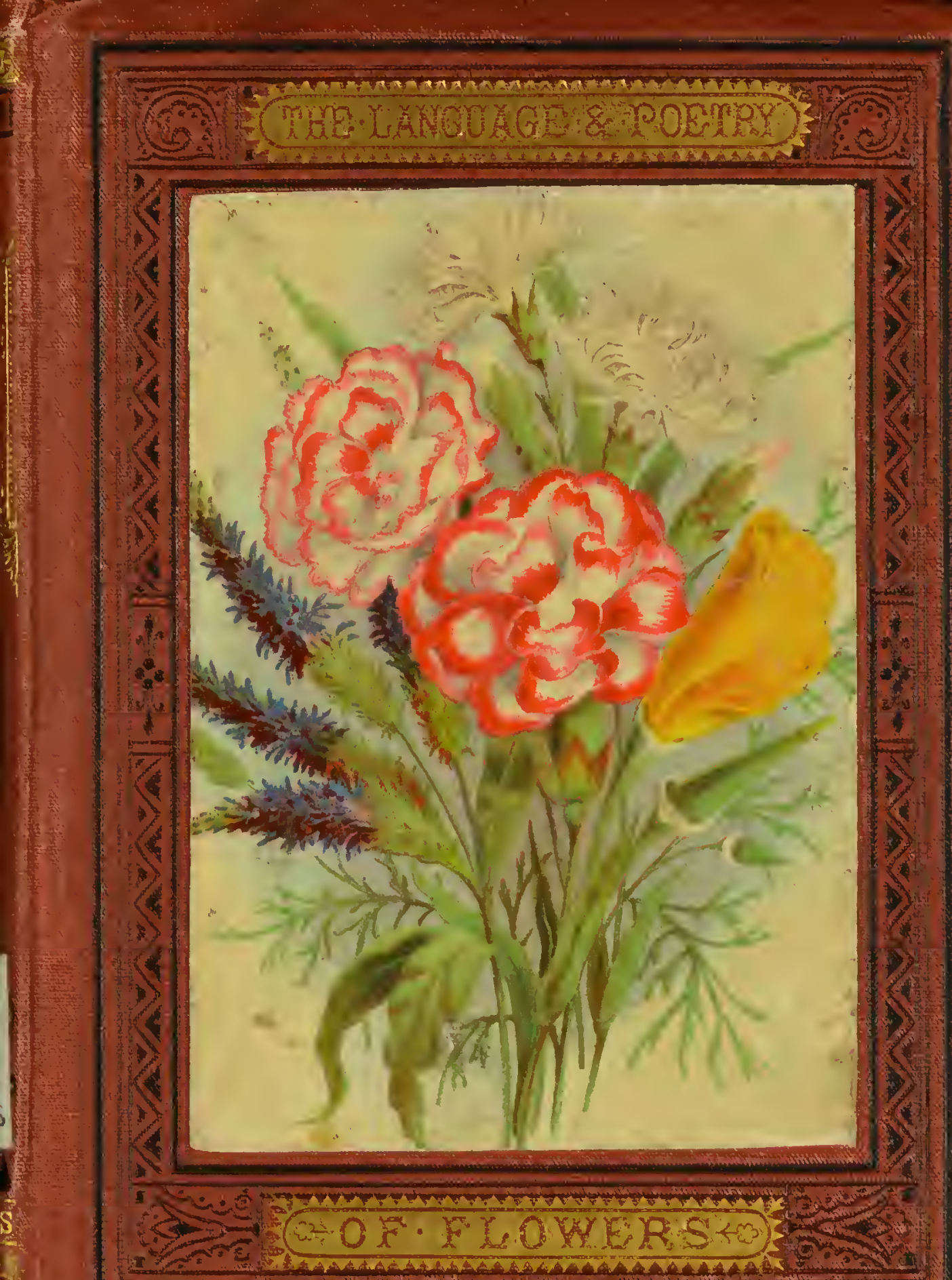




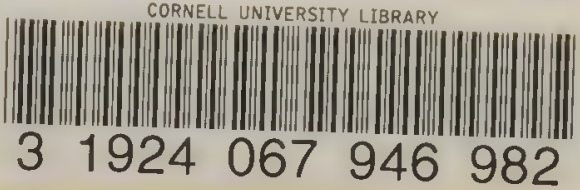

DATE DUE

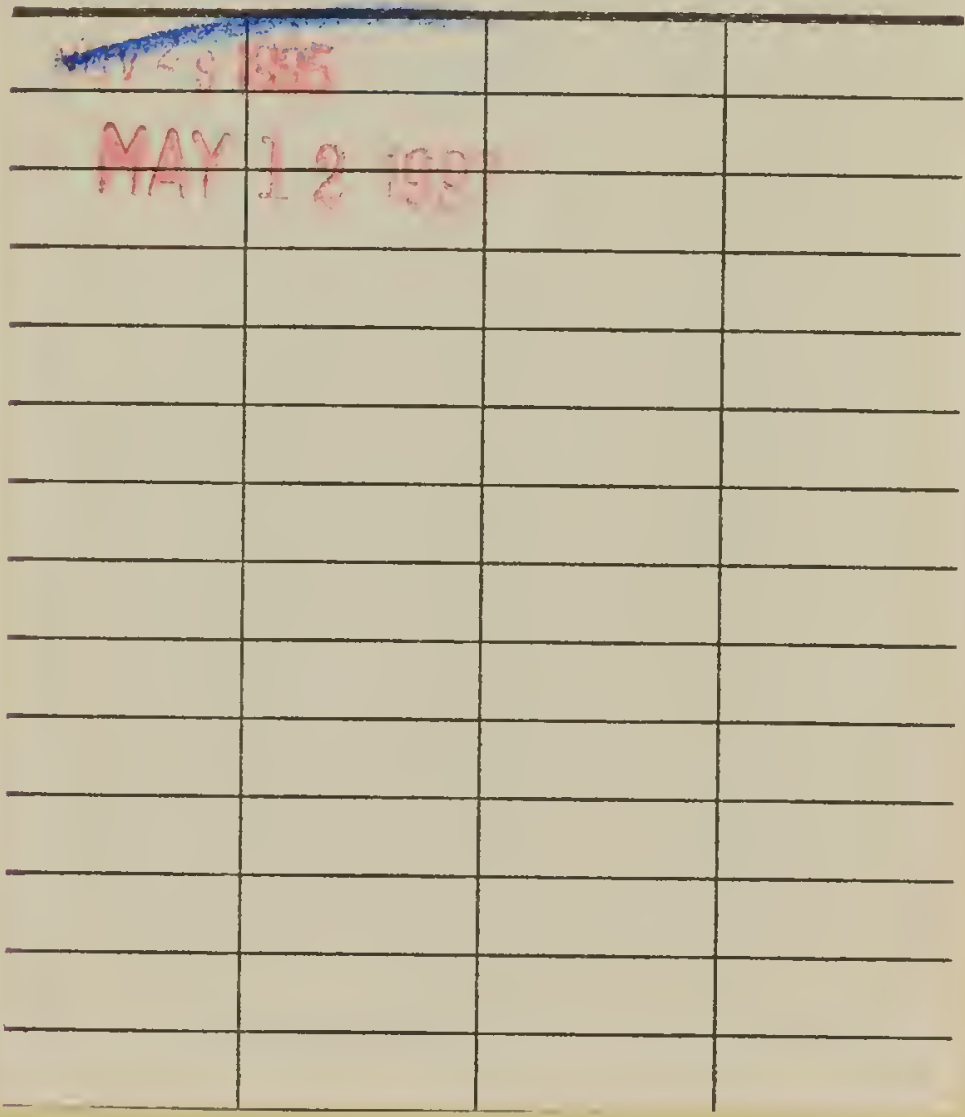




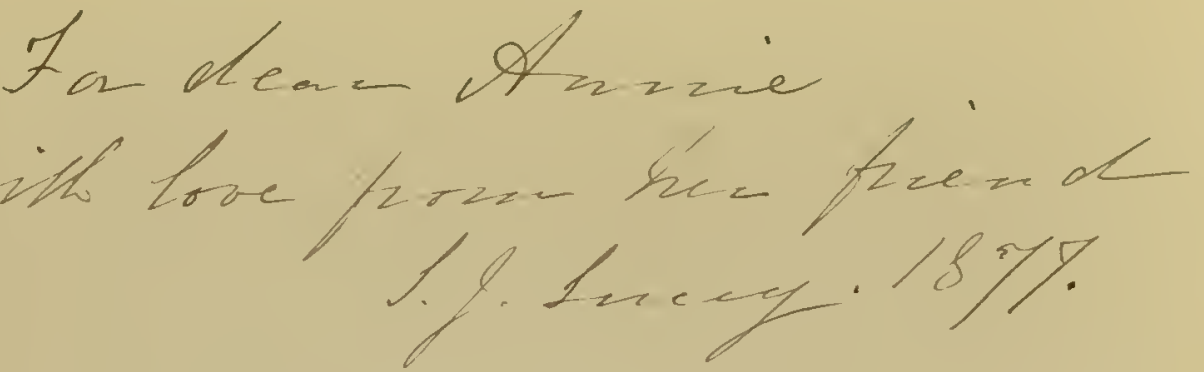

\section{THE LANGUAGE OF FLOWERS.}

Flowers are the bright remembrancers of youth;

They waft us back, with their bland odorous breath, The joyous hours that only young life knows,

Ere we have learned that this fair earth hides graves. 




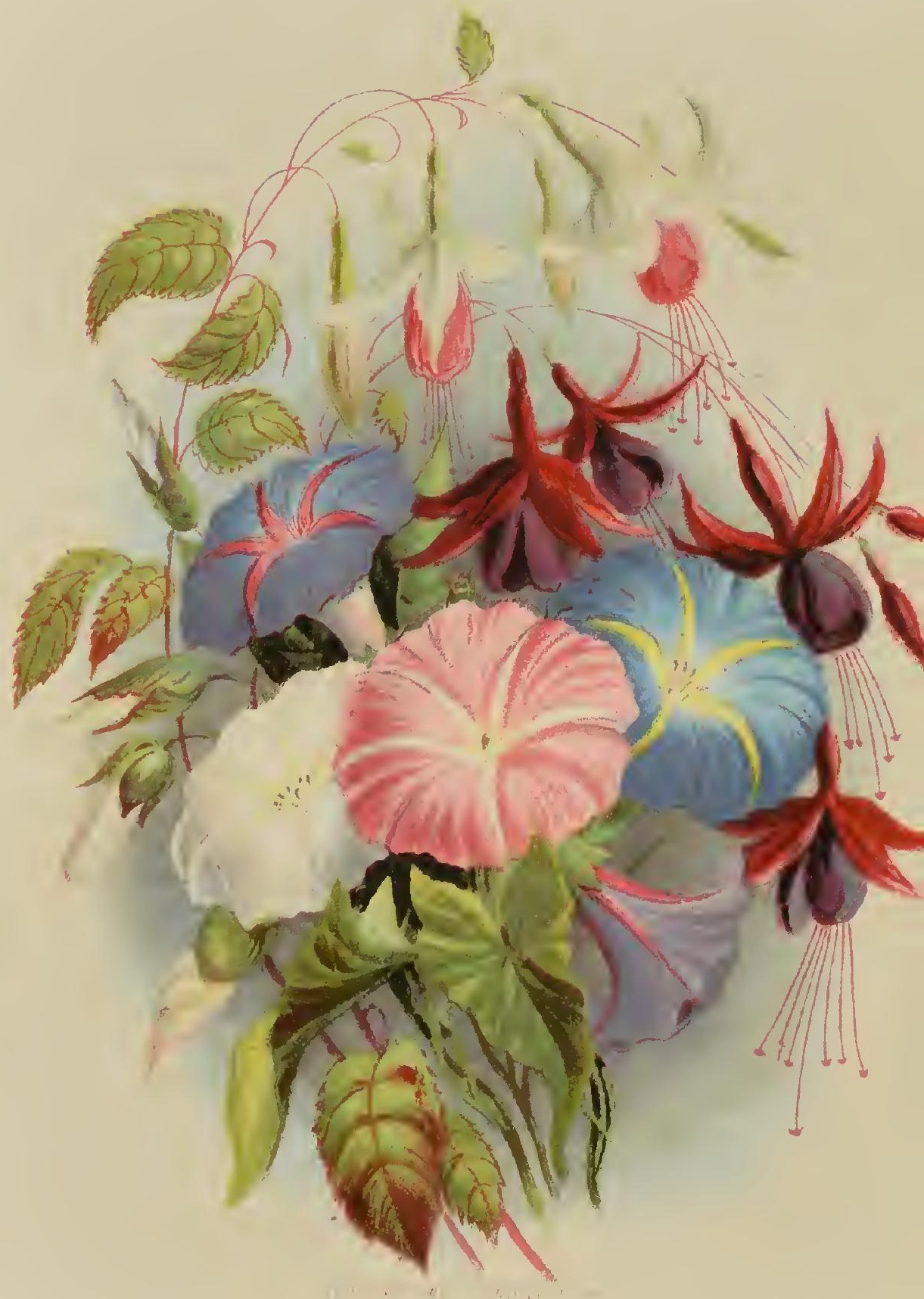




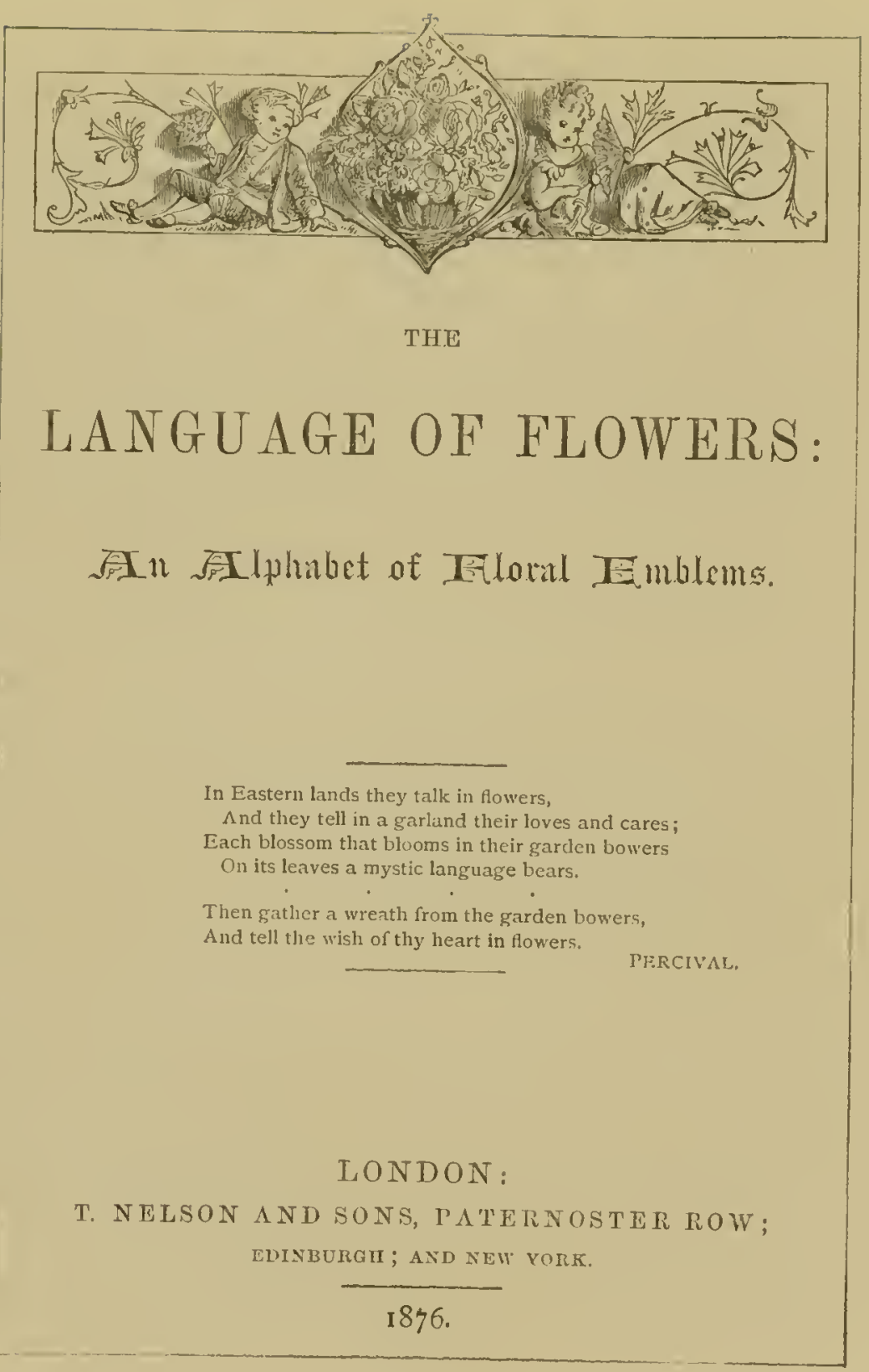


VAULT

GR

780

132

1776 


\section{Contents.}

$\begin{array}{llllllr}\text { INTRODUCTION, } & \ldots & \ldots & \ldots & \ldots & \ldots & 7 \\ \text { DIAL OF FLOWERS, } & \ldots & \ldots & \ldots & \ldots & \ldots & 11 \\ \text { THE FLOWER-DIAL, } & \ldots & \ldots & \ldots & \ldots & \ldots & 12\end{array}$

PART I.

FLOWERS, AND THE SENTIMENTS WHICH THEY REPRESENT,

PART II.

SENTIMENTS, AND THE FLOWERS WHICH REPRESENT THEM,

THE POETRY OF FLOWERS-

The Use of Flowers, Acacia, Ycllow, ... Amaranth, Balsam, ... Blue Bell, Camomile, Canterbury Bell, Carnation, Yellow, ... Catchfly, Red, China Aster, Double, Clematis, Cowslip, ... Dahlia, Eglantine,

$\begin{array}{lllll}\ldots & \ldots & \ldots & \ldots & 67 \\ \ldots & \ldots & \ldots & \ldots & 69 \\ \ldots & \ldots & \ldots & \ldots & 69 \\ \ldots & \ldots & \ldots & \ldots & 70 \\ \ldots & \ldots & \ldots & \ldots & 70 \\ \ldots & \ldots & \ldots & \ldots & 71 \\ \ldots & \ldots & \ldots & \ldots & 72 \\ \ldots & \ldots & \ldots & \ldots & 72 \\ \ldots & \ldots & \ldots & \ldots & 73 \\ \ldots & \ldots & \ldots & \ldots & 74 \\ \ldots & \ldots & \ldots & \ldots & 74 \\ \ldots & \ldots & \ldots & \ldots & 75 \\ \ldots & \ldots & \ldots & \ldots & 76 \\ \ldots & \ldots & \ldots & \ldots & 77\end{array}$


Forget-me-not,

Gentian, ...

Geranium, Scented,

Gilly Flower,

Hawthorn,

Honeysuckle, Wild,

Hydrangea,

Iris,

Jasmine, TWhite, ...

Jasmine, Yellow, ...

Jonquil,

Larkspur, Pink,

Lilac, Purple,

Lily, White,

London Pride,

Mignonette,

Pansy,

Passion Flower,

Pea, Everlasting, ...

Pea, Sweet,

Peach Blossom,

Phlox,

Pink, Carnation,

Polyanthus,

Primrose, Evening,

Rose, Bridal,

Rosebud, Moss

Rose, Deep Red,

Rose, Full Red,

Sunflower, Dwarf, ..

Sweetwilliam,

Syringa,

Tulip, Variegated, ...

Tulip, Yellow,

Verbena,

Veroni ia,

Violet, Blue,

Violet, Purple,

Wallflower,

TVoodruff,

Hymn to the Flowers,

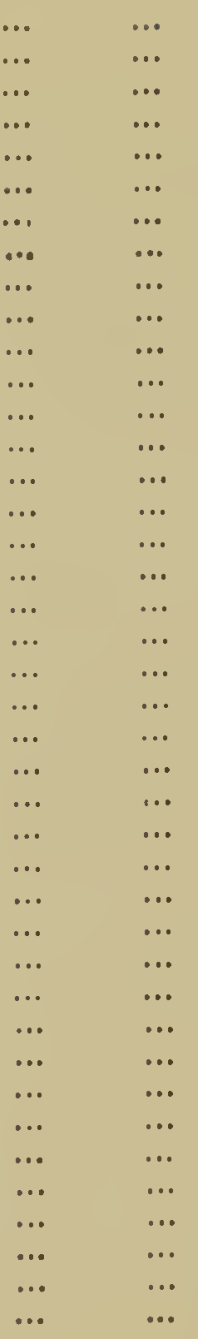

... 


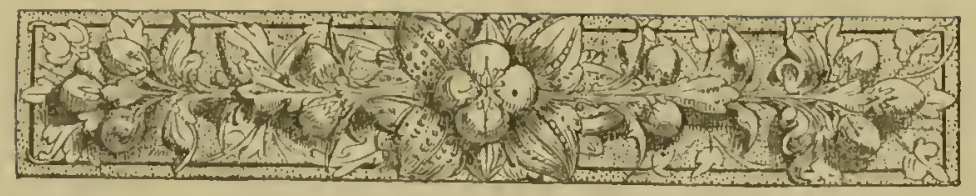

\section{可entroduction.}

HE Language of Flora has been traced by its students to widely different sources, each presenting some true claim to the title, yet none so entirely subverting those of others as to stand forth alone as its originator; for truly the origin of this voice of the flowers is coeval with their creation, and is still a tongue sufficiently simple and attractive to have a charm for every student of Nature, and to suggest appropriate emblems even to the illiterate rustic, who plucks the wayside daisy or the blue forget-me-not, to be presented to some village maiden as the readiest expression of liis love.

It is, in truth, no creature of modern art, but the freeborn child of unsophisticated nature. "Lovely as the rose," "Fair as the lily," or "Modest as the violet," are 
phrases that seem to come naturally into use, without thought that in this emblematic employment lies the germ of true poetry and the symbolic language of Flora; and though to these will be found added, in the present volume, many wherein the object seems less suggestive of the sense, and where the idea sought to be conveyed is more complex and difficult intelligently to symbolize, yet in this is only presented the floral tongue passing through the same progressive stages that have characterized the annals of every spoken language. In a rude and primitive state, the words are few and simple that suffice to clothe in language the thoughts and desires of an untutored race of men; but with every increasing want, and every new desire, names and forms of thought must be created, until the brief vocabulary of the savage tribe swells into the complex dictionary of an intelligent and civilized people. And so has it been with this universal language. "He cometh forth as a flower and is cut down," is the expressive and universally intelligible language of Scripture. And no less does it early prefigure hope than frailty. We strew them over the shroud of departed love, and plant them to bloom brightly above the grave, that they may speak in spring of a brighter season of hope,-and in summer of that heavenly clime that knows only of an eterual summer and a cloudless sky, 
-and in all seasons, of love, and purity, and peace. To these, the simple expressions of natural feeling, have been added from time to time, from the pages of classic poetry and the more complex fancies of later writers, a series of ideas attached to every flower, by means of which the nosegay may be made to take the place of more formal epistles.

For the more complicated uses of this beautiful language, a few hints may be necessary to show how extensive is the range of thought its alphabet may communicate. For example: if a flower be given reversed, it implies the opposite of that thought or sentiment which it is ordinarily understood to express; again, a rosebud from which the thorns have been removed, but which has still its leaves, conveys the sentiment, "I fear, but I hope,"-the thorns implying fear, as the leaves hope; remove the leaves and thorns, and then it signifies, "There may be neither hope nor fear;"-while, again, a single flower may be made emblematical of a variety of ideas: a rosebud that has been already used and deprived of its thorns, says, "There is much to hope;" but stripped of its leaves also, it tells, "There is everything to fear." The expression, also, of almost any flower may be varied by changing its position. Place the marigold upon the head, for instance, and it signifies "distress of mind ;" on the bosom, "listlessness." And, it may be added, when a flower is given, the pronoun 
$I$ is understood by clianging it to the right, thou by in clining it to the left.

To the intelligent reader, these suggestions may suffice; and we shall therefore conclude our introduction with the liope that, under the guidance of this little volume, many a bright nosegay may exchange hands, and tell, in its titting and intelligible language, "a welcome message to fair lady's ear."

In eastern lands they talk in flowers, And they tell in a garland their loves and cares;

Each blossom that blooms in their garden bowers

On its leaves a mystic language bears.

The rose is a sign of joy and love,

Young blushing love iu its earliest dawn;

And the mildness that suits the gentle dove,

From the myrtle's snowy flower is drawn.

Innocence shines in the lily's bell,

Pure as the heart in its native heaven:

Fame's bright star and glory's swell,

By the glossy leaf of the bay are given.

The silent, soft, and humble heart,

In the violet's hidden sweetness breathes;

And the tender soul that cannot part,

A twine of evergreen fondly wreathes.

'The cypress that daily shades the grave,

Is sorrow that mourns her bitter lot; And faith, that a thousand ills can brave,

Speaks in thy blue leaves-forget-me-not.

Theu, gather a wreath from the garden bowers, And tell the wish of thy heart in flowers.

Perctyal. 


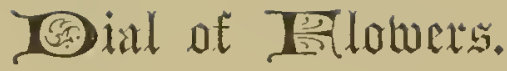

TIME OF OPHNINA.

u. м.

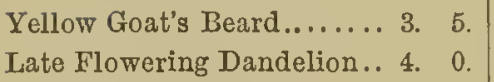

Bristly Helminthia........ 4. 5 .

Alpine Borkhausia......... 4. 5.

Wild Succory............. 4. 5 .

Naked-stalked Poppy ....... 5. 0.

Copper-coloured Day Lily .. 5. 0.

Smooth Sow Thistle....... 5. 0.

Small Bindweed........... 5. 6.

Common Nipple Wort...... 5. 5.6.

Common Dandelion........ 5. 6.

Spotted Achyrophorus...... 6. 7 .

White Water-Lily......... 7. 0.

Garden Lettuce ............ 7. 0.

African Marigold ......... 7. 0.

Common Pimpernel....... 7. 8.

Mouse-ear Hawkweed ...... 8. 0.

Proliferous Pink........... 8. 0.

Field MIarigold. ........... $0 . \quad 0$.

Purple Sandwort... . . . . . . . 0. 10.

Small Purslane............ 0. 10.

Creeping Mallow......... 0. 10.

Clickweed.............. 9. 10.
TIME OF CLOSINA.

II. 31. Bristly Helminthia.........12. 0 . Alpine Borkhausia.........12. 1. Late Flowering Dandelion ...12. 0 . Creeping Mallow ...........12. 1 . Proliferous Pink........... 1. 0 . Mouse-ear Hawkweed ...... 2. 0. Common Pimpernel........ 2. 3. Purple Sandwort........... 2. 3. Field Marigold. ........... 3. 0 . African Marigold .......... 3. 4. Small Bindweed ........... 4. 5 . Spotted Achyrophorus ...... 4. 5. White Water-Lily.......... 5. 0. Naked-stalked Poppy....... 7. 0. Copper-coloured Day Lily .. 7. 8. Wild Succory .............. 8. 9. Common Dandelion........ 8. 9. Yellow Goat's Beard......... 9. 10. Chickweed................9. 10. Common Nipple Wort.......10. 0. Garden Lettuce... . . . . . . . . 10. 0 . Small Sow Thistle..........11. 12. Small Purslane............11. 12 . 


\section{周he Tlotwer-Tial.}

'Twas a lovely thought, to mark the hours,

As they floated in light away,

By the opening and the folding flowers,

That laugh to the summer's day.

Thus had each moment its own rich hue, And its graceful cup and bell,

In whose coloured vase might sleep the dew,

Like the pearl in an ocean-shell.

To such sweet signs might the time have flowed

In a golden current on,

Ere from the garden, man's first abode,

The glorious guests were gone.

So might the days have been brightly told-

Those days of songs and dreams, -

When shepherds gathered their flocks of old

By the blue Arcadian streams.

So in those isles of delight that rest

Far off in a breezeless main,

Which many a bark, witl a weary guest,

Has sought, but still in vain.

Yet is not life, in its real flight,

Marked thus --even thus-on earth,

By the closing of one hope's delight,

And another's gentle birth ?

oh ! let us live so that flower by flower,

Shutting in turn, may leave

A lingerer still for the sunset hour-

A charm for the shaded eve.

HEMANS. 


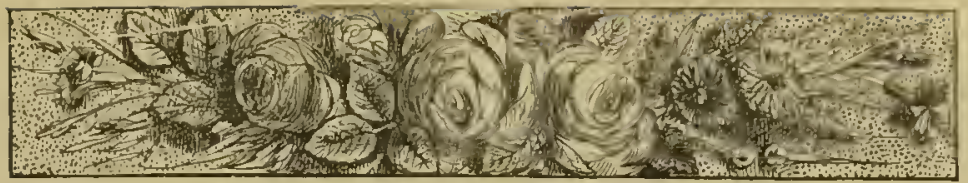

\section{THE LANGUAGE OF FLOWERS.}

\section{PART FIRST.}

dlowers, and the Sentiments which then firpresent.

Acacia........................Chaste love.

Acacia, Pink.................Elegance.

Acacia, Rose..................Platonic love. Friendship.

Acacia, Yellow...............Secret love.

Acanthus..................... The Fine Arts. Artifice.

Adonis........................ Sorrowful remembrances.

Agnus Castus.................Indifference. Coldness.

Agrimony....................Thankfulness.

Almond Tree................Stupidity. Indiscretion.

Aloe...........................Afliction. Grief.

Althæa Frutex..............Persuasion.

Amaranth................... Immortality.

Amaranth, Globe............Unchangeable. 
Amaryllis...................Timidity. Pride.

Ambrosia.......................Love returned.

Anemone, Field..............Sickness.

Anemone, Garden...........Forsaken.

Angelica ..................... Inspiration.

Angrec......................Royalty.

Apocynum...................Deceit.

Apple.......................Temptation.

Apple Blossom...............Preference.

Apple, Pine.................Perfection.

Apple, Thorn................ Deceitful charms.

Arbor Vitæ..................Unchanging friendship.

Arum (Wake Robin).........Ardour.

Ash, Mountain..............Prudence.

Ash Tree.....................Grandeur.

Aspen Tree..................Lamentation.

Asphodel......................My regrets follow you to the

grave.

Auricula ....................Painting.

Auricula, Scarlet............Avarice.

Azalea .......................Temperance.

Balm.......................Sympathy.

Balm, Gentle ................Pleasantry.

Balm of Gilead...............Cure. Retief.

Balsam ....................... Impatience. Ardent love. 
Barberry....................Sourness. Sharpmess. Ill temper.

Basil, Sweet................Ilatred.

Bay Leaf....................I change but in death.

Bay Tree...................Glory.

Bay Wreath................Reward of merit.

Bearded Crepis..............Protection.

Beech Tree..................Prosperity.

Bee Ophrys ..................Error.

Bee Orchis................. Industry.

Belladonna....................Silence.

Bell Flower, White..........Gratitude.

Belvedere....................I declare against you.

Betony ...................... Surprise.

Bilberry....................Treachery.

Birch Tree....................Meekness.

Bindweed, Great............. Insinuation.

Bindweed, Small............IIumitity.

Bladder Nut Tree...........Amusement. Frivolity.

Blaeberry .................... Ingenuous simplicity.

Bluebell.....................Constancy.

Bluebottle (Centaury).......Delicacy.

Bonus Henricus.............Goodness.

Borage ...................... Bluntness.

Box........................Stoicism. 
Bramble....................Lowliness. Envy. Remorse. Broom .......................Neatness. Humility.

Buckbean .....................Calm. Repose.

Bugloss.......................Falsehood.

Bulrush...................... Docitity.

Burdock......................Touch me not. Importunity.

Buttercup (Kingcup).........Childishness. Ingratitude.

Butterfly Orchis...............Gaiety.

Cabbage.....................Gain. Profit.

Calla Ethiopica...............Magnificent beauty.

Calycanthus..................Compassion. Benevolence.

Camellia Japonica............Unpretending excellence.

Camomile.....................Energy in adversity.

Canary Grass ................Perseverance.

Candytuft..................... Indifference.

Canterbury Bell..............Acknowledgment.

Cardamine...................Paternal error.

Cardinal Flower..............Distinction.

Carnation ....................Fascination. Woman's love.

Carnation, Striped...........Refusal.

Carnation, Yellow ............Disdain.

Catchfly.....................Pretended love. Snare.

Catchfly, Red................ Youthful love.

Catchfly, White..............Betrayed.

Cedar Leaf ..................I live for thee. 
Cedar of Lebanon........... Incorruptible.

Cedar Tree...................Strength. Constancy.

Celandine .....................Joys to come.

Centaury .....................Felicity.

Champignon..................S'uspicion.

Cherry Tree.................Education.

Cherry Tree, White.........Deception.

Chestnut.....................Luxury.

Chestnut Tree.................Do me justice.

Chickweed....................Rendezvous.

Chickweed, Mouse-eared....Ingenuous simplicity.

Chicory .......................Frugality.

China Aster................. Variety.

China Aster, Double........I partake your sentiments.

China Aster, Single.........I will think of $i$ t.

Chrysanthemum, Chinese...Cheerfulness under misfortune.

Chrysanthemum, Red.......I love.

Chrysanthemum, White.....Truth.

Chrysanthemum, Yellow...Slighted love.

Cinquefoil..................... Maternal affection.

Cistus, Gum..................I shall die to-morrow.

Clematis......................Mental beauty.

Clematis, Evergreen .........Poverty.

Clotbur......................Rudeness.

(499) 
Clover, Red..................Industry.

Cloves ....................... Dignity.

Cobæa........................Gossip.

Coltsfoot...................... Justice shall be done.

Columbine.................... Folly.

Columbine, Purple...........Resolution.

Columbine, Red..............Anxious and trembling.

Convolvulus..................Bonds. Uncertainty.

Convolvulus, Major..........Extinguished hope.

Convolvulus, Minor.......... Night.

Coreopsis......................Always cheerful.

Coreopsis, Arkansa.......... Love at first sight.

Coriander .....................Concealed merit.

Corn...........................Riches.

Cornbottle....................Delicacy.

Coronilla.......................Success crown your wishes.

Cowslip......................Pensiveness. Winning grace.

Cowslip, American........... You are my divinity.

Cranberry.....................Cure for heartache.

Crane's Bill................... Envy.

Cresses.........................Stability.

Crocus........................Abuse not.

Crocus, Spring................ Youthful gladness.

Crown Irnperial............... Mrajesty.

Cucumber.

Criticism. 
Cudweed......................Never-ceasing remembrance.

Currants..................... You please all.

Cuscuta........................Mreanness.

Cypress....................Death. Mourning.

Cypress and Marigold.......Despair. Melancholy.

Daffodil......................Regard.

Daffodil, Great Yellow......Chivalry.

Dahlia..................... Instability.

Daisy, Double...............Participation.

Daisy, Garden ..............I share your sentiments.

Daisy, Ox Eye..............A token.

Daisy, Party-coloured ........Beauty.

Daisy, Red ..................Unconscious.

Daisy, White ................Innocence.

Daisy, Wild.................I witl think of it.

Dandelion.....................Love's Oracle.

Daphne Odora...............Painting the Lity.

Darnel........................ Vice.

Dead Leaves...................Sadness.

Dew Plant................... serenade.

Diosma .......................Uselessness.

Dittany of Crete.............Birth.

Dittany, White .............Passion.

Dock ........................Patience.

Dodder........................Ireanness. 
Dodder of Thyme ........... Business. Care. Selfishness.

Dogsbane....................Deceit. Falsehood.

Dogwood .....................Duration.

Dogwood Blossom...........Am I perfectly indifferent to you?

Dragon Plant................ Snare.

Dragonwort .................Horror.

Ebony........................Blackness. You are hard. Eglantine (or Sweet Brier).Poetry. I wound to heal. Elder...........................Zealousness. Compassion.

Elm ......................... Dignity.

Endive........................Frugality.

Eupatorium ..................Delay.

Fennel.......................Force. Strength.

Fern.........................Sincerity.

Fern, Flowering..............Reverie.

Fever Root...................Delay.

Ficoides...................... Your looks freeze me.

Fig ............................Argument. Longevity.

Fig Tree .....................Profuseness.

Filbert.......................Reconciliation.

Fir of Gilead .................Juice.

Fir Tree......................Elevation.

Flax (Linum)................I feel your kindness. Donestic industry. 
Fleur-de-lis.................Flane.

Flora's Bell.................. You make no pretension.

Flowering Reed.............. Confudence in Heaven.

Flower-of-an-hour........... Delicate beauty.

Forget-me-not...............Remembrance. True love. Forget me not.

Foxglove....................Insincerity. A wish.

Frankincense ............... The incense of a faithful heart.

Fritillary, Chequered.........Persecution.

Frog Ophrys................ Disgust.

Fuchsia, Scarlet.............Taste.

Fuller's Teasel............... Importunity. Misanthromy.

Fumitory....................Spleen.

Geranium ...................Gentility.

Geranium, Apple...........Present preference.

Geranium, Dark..............Melancholy.

Geranium, Fish.............Disappointed expectation.

Geranium, Ivy...............I engage you for the next dance.

Geranium, Nutmeg.........An expected meeting.

Geranium, Oak..............Lady, deign to smile.

Geranium, Pencil-leaved....Ingenuity.

Geranium, Rose or Pink....Preference.

Geranium, Scarlet..........Comforting. Stupidity.

Geranium, Silver-leaved....Recall.

Gerauium, Sorrowful......... Melancholy mind. 
Geranium, Wild.............Steadfast piety.

Gilly Flower.................Unfading beauty. Bonds of affection.

Goat's Rue...................Reason.

Golden Rod..................Precaution. Encouragement.

Gooseberry ...................Anticipation.

Gorse........................Enduring affection.

Gourd........................Extent. Bulk.

Grass........................Utility. Submission.

Harebell....................Grief. Submission.

Hawkweed ................... Quick-sightedness.

Hawthorn....................Hope.

Hazel .........................Reconciliation.

Heath........................ Sotitude.

Helenium ....................Tears.

Heliotrope...................Devotion. Faithfulness.

Hellebore.....................Scandal. Calumny.

Hemlock..................... You will be my death.

Hemp.......................Fate.

Henbane....................Defect.

Hepatica .....................Confidence.

Hibiscus..................... Delicate beauty.

Holly........................Foresight. Am I forgotten?

Holly Herb..................Enchantment.

Hollyhock..................Fecundity. 
Hollyhock, White...........Female ambition.

Honesty......................Honesty. Sincerity.

Honey Flower............... Love, sweet and secret.

Honeysuckle, Coral..........The colour of my fate.

Honeysuckle, French.........Rustic beauty.

Honeysuckle, Monthly......Bond of love. Domestic happiness. I will not answer hastily.

Honeysuckle, Wild.......... Inconstancy in love.

Hop.........................Injustice.

Horehound...................Fire.

Hornbeam Tree.............. Ornament.

Hortensia .................... You are cold.

Houseleek................... Vivacity. Domestic economy.

Houstonia...................Content.

Hoya........................Sculpture.

Hyacinth.....................Sport. Play.

Hyacinth, Blue..............Constancy.

Hyacinth, Purple............Sorrow.

Hydrangea..................A Boaster. Heartlessness.

Hyssop.......................Cleanliness.

Ice Plant.....................Rejected addresses.

Indian Cress................Eclat. Warlike traphy.

Indian Plum.................Privation.

Ipomœa.....................Attachment. 
Tris Ify compliments. I have a message for you.

Iris, Yellow..................Flame. Passion. Ivy .........................Friendship. Fidelity. Matrimony.

Ivy sprig, with tendrils......Assiduous to please. Jasmine, Cape ...............Transport of joy. Jasmine, Carolina............Separation. Jasmine, Indian..............I attach myself to you. Jasmine, Spanish ............Sensuatity. Jasmine, White..............Amiabitity. Jasmine, Yellow.............. Grace and elegance. Jonquil........................ I desire a return of affection. Judas Tree..................Unbelief. Betrayal. Juniper........................Succour. Protection. Justicia ..................... The perfection of female loveliness.

Kennedya....................Mental beauty.

Kingcup......................I wish I were rich.

Laburnum...................Forsaken. Pensive beauty.

Lady's Mantle...............Fashion.

Lady's Slipper...............Fickleness. Capricious beauty. Lagerstræmia.................Eloquence.

Lantana.....................Rigour.

Larch........................Audacity. Boldness. 
Larkspur ....................Lightness. Levity.

Larkspur, Double.............Ilaughtiness.

Larkspur, Pink..............Fickleness.

Laurel, Common ............Perfidy.

Laurel, Mountain............Ambition. Glory.

Laurestina....................A token. I die if neglected.

Lavender.....................Distrust.

Lavender, Sea................Dauntlessness.

Lemon.......................Zest.

Lemon Blossoms..............Fidelity in love. Discretion.

Lettuce........................Cold-hearted.

Lichen....................... Dejection. Solitude.

Liquorice, Wild .............I declare against you.

Lilac, Field ..................IIumitity.

Lilac, Purple.................The first emotions of love.

Lilac, White..................Purity. Modesty. Youthful

Innocence.

Lily, Day....................Coquetry.

Lily, Imperial.................Mrajesty.

Lily of the Valley............Return of happiness.

Lily, White...................Purity and sweetness.

Lily, Yellow..................Falsehood. Gaiety.

Linden (Lime Tree).......... Conjugal love.

Lint.......................... I feel all my obligations.

Liverwort................... Confidence. 
Lobelia.......................Malevolence.

Locust Tree...................Elegance.

Locust Tree, Green...........Affection beyond the grave.

London Pride................Frivolity.

Lotus.........................Eloquence.

Lotus Flower................Estranged love.

Lotus Leaf ..................Recantation.

Love-in-a-Mist..............Perplexity.

Love-in-a-Puzzle..............Embarrassment.

Love-lies-bleeding............Hopeless, not heartless.

Lucerne......................tife.

Lupine...................... Voraciousness.

Lychnis....................A religious enthusiast.

Lychnis, Meadow............Wit.

Lychnis, Scarlet..............Sun-beamed eyes.

Lythrum....................Pretension.

Madder......................Calumny.

Magnolia..................... Love of nature.

Magnolia, Swamp...........Perseverance.

Maiden Hair ................ Discretion.

Maidwort...................Tranquillity.

Mallow, Marsh ..............Beneficence.

Mallow, Syrian...............Consumed by love.

Mallow, Venetian............Delicate beauty.

Manchineal Tree.............Hypocrisy. Falsehood. 
Mandrake...................Horror.

Maple.......................Reserve.

Marigold.....................Grief.

Marigold, African........... Vulgar-minded.

Marigold, French.............Jealousy.

Marjoram....................Blushes.

Marvel of Peru..............Tinidity.

Meadow Saffron..............My best days are past.

Meadowsweet.................Uselessness.

Mesembryanthemum.........Idleness.

Mezerion.....................I desire to please.

Mignonnette.................Your quatities surpass your Milfoil.......................War. [charms.

Milkvetch.................... Your presence softens my pain.

Milkwort.....................Hermitage.

Mimosa (Sensitive Plant)...Sensitiveness.

Mint......................... Virtue.

Mistletoe ......................I surmount all obstacles.

Mock Orange................ Counterfeit.

Moonwort...................Forgetfulness.

Moschatel..................... Weakness.

Moss.......................... Maternal love.

Moss, Iceland.................Health.

Mosses ........................Ennui.

Motherwort................. Secret love. 
Mourning Bride............. Unfortunateattachment. I have lost all.

Moving Plant. Agitation.

Mugwort.....................ITappiness.

Mulberry, Black............I will not survive you.

Mulberry Tree............... Wisdom.

Mullen.......................Good-nature.

Mushroom...................Suspicion.

Musk, Crowfoot..............Weakness.

Mustard Seed.................Indifference.

Myrobalan...................Privation.

Myrrh.......................Gladness.

Myrtle.........................Love.

Narcissus, Poet's.............Egotism. Self-esteem.

Nasturtium..................Patriotism. Warlike trophy.

Nasturtium, Scarlet.........Splendour.

Nettle.......................Cruelty.

Nettle, Burning ..............Slander.

Nettle Tree..................Concert. Plan.

Night-blooming Cereus.....Transient beauty.

Night Convolvulus...........Night.

Nightshade...................Sorcery. Scepticism. Witchcraft. Dark thoughts.

Nightshade, Bitter...........Truth.

Nosegay, a..................Gallantry. 
Oak Leaf....................Bravery and humanity.

Oak Tree.....................IIospitality.

Oats.........................Music.

Oleander ....................Beware.

Olive Branch.................Peace.

Orange.......................Generosity.

Orange Blossom.............. Your purity equals your loveliness.

Orchis.........................A beauty. A belle.

Osier..........................Frankness.

Osmunda ......................Dreams.

Ox-Eye ......................Patience.

Palm ......................... Victory.

Pansy (Heart's-ease)......... You occupy my thoughts.

Parsley.......................Feasting. Useful knowledge.

Pasque Flower................ You have no claims.

Passion Flower...............Belief. Susceptibility. Re-

ligious superstition.

Peach Blossom ................I am your captive.

Pea, Everlasting..............An appointed meeting. Lasting pleasure.

Pea, Sweet ...................Departure.

Pear, Prickly ..................Satire.

Pear Tree.....................Comfort.

Pennyroyal....................Flee away. 
Peony.......................Shame. Bashfulness.

Pepppermint................Warmth. Cordiality.

Periwinkle, Blue............Pleasures of memory.

Periwinkle, Red..............Early friendship.

Periwinkle, White...........Pleasant recollections.

Persicaria...................Restoration.

Persimmon ...................Bury me amid nature's beauties.

Phlox....................... Unanimity.

Pine, Black..................Pity.

Pine, Pitch.................Time. Philosophy.

Pine, Spruce ................Farewell. Hope in adversity.

Pink..........................Boldness.

Pink, Carnation.............. Woman's love.

Pink, Indian Double.........Always lovely.

Pink, Indian Single..........Aversion.

Pink, Mountain............. You are aspiring.

Pink, Red Double........... Pure and ardent love.

Pink, Variegated.............Refusal.

Pink, White.................Ingeniousness. Talent.

Pimpernel....................Change.

Plane Tree ................... Genius.

Pleurisy Root ................Cure for heartache.

Plum Tree...................Perform your promise.

Plum Tree, Wild.............Independence.

Polyanthus..................Pride of riches. 
Polyanthus, Crimson.........The heart's mystery.

Polyanthus, Lilac............Confidence.

Pomegranate.................Footishness.

Pomegranate Flower.........Mature elegance.

Pomme Rose (Rose-Apple)..Gentility. Prettiness.

Poplar, Black...............Courage.

Poplar, White................Time.

Poppy .......................Evanescent pleasure.

Poppy, Red..................Consolation.

Poppy, Scarlet...............Fantastic extravagance.

Poppy, White................Sleep. My bane, my antidote.

Potato........................Benevolence.

Pride of China................ Dissension.

Primrose.....................Early youth.

Primrose, Evening ........... Inconstancy.

Primrose, Red................ Unpatronised merit.

Privet.........................Prohibition.

Quaking Grass................Agitation.

Quamoclit....................Busybody.

Queen's Rocket..............F Fashionable. Fou are the queen of coquettes.

Quince.......................Temptation.

Ragged Robin.................Wit.

Ranunculus................... am dazzled by your charms.

Ranunculus, Garden......... You are rich in attractions. 
Ranunculus, Wild...........Ingratitude.

Raspberry ...................Remorse.

Ray Grass ................... Vice.

Red Bay .....................Love. Memory.

Reed........................Complacence.

Reeds, Split .................Indiscretion.

Rest Harrow................. Obstacle.

Rhododendron ...............Danger.

Rhubarb....................Advice.

Rocket......................Rivalry.

Rose ...........................Love.

Rose, Austrian............... Thou art all that is lovely.

Rose, Bridal..................Happy love.

Rose, Burgundy ............... Unconscious beauty.

Rose, Cabbage................Ambassador of love.

Rose, Campion................ Only deserve my love.

Rose, Carolina................ Love is dangerous.

Rose, China................... Beauty always new.

Rose, Christmas..............Tranquillize my anxiety.

Rose, Daily ...................Thy smile I aspire to.

Rose, Damask................Brilliant complexion.

Rose, Deep Red..............Bashful shame.

Rose, Dog...................Pleasure and pain.

Rose, Full - blown, placed

over two buds...............Secrecy. 
Rose, Full Moss.............. Superior merit.

Rose, Full Red...............Beauty.

Rose, Full White...........I ain worthy of you.

Rose, Guelder................ Winter of age.

Rose, Hundred-leaved.......Pride.

Rose, Japan .................. Beauty is your only attraction.

Rose, Lancaster..............Union.

Rose, Maiden-blush.......... If you love me you will discover it.

Rose, May...................Precocity.

Rose, Mundi................ Variety. Tou are merry.

Rose, Musk..................Capricious beauty.

Rose, Musk, Cluster.........Charming.

Rose, Red-leaved............. Beauty and prosperity.

Rose, Thormless ..............Early attachment.

Rose, Unique................Call me not beautiful.

Rose, White and Red, together......................Unity.

Rose, Withered White......Traisient impression.

Rose, Yellow.................Jealousy. Decrease of love.

Rose, York.................War.

Roses, Crown made of.......Reward of virtue.

Rosebud, Moss ..............Confession of love.

Rosebud, Red................ You are young and beautiful.

Rosebud, White..............A heart ignorant of love. 
Rosebay.....................Beware.

Rosemary.................... Your presence revives me. liemembrance.

Rudbeckia....................Justice.

Rue.........................Disdain.

Rush.........................Docility.

Saffron.......................Marriage.

Saffrom, Crocus...............Mirth.

Suffron, Flower...............Do not abuse. Beware of excess.

Sage.........................Esteem. Domestic virtues.

Satin Flower................Sincerity.

Scabious..................... Unfortunate love.

Scabious, Sweet.............. Widowhood.

Senvy.......................Indifference.

Service Tree..................Prudence.

Shamrock.....................Light-heartedness. Emblem of Ireland.

Snakesfoot...................Horror.

Suake's Lounge..............Slander.

Snapdragon..................Presumption.

Snowball....................Bound.

Snowball Tree................Age.

Snowdrop...................Consolation. Hope.

Sorrel........................ I'arental affection.

Sorrel, Wild..................Wit, ill-timed. 
Southernwood.................Jest. Bantering.

Sowbread.....................Diffidence.

Spearmint....................Warmth of sentiment.

Speedwell.....................F emale fidelity.

Speedwell, Germander......Facility.

Speedwell, Spiked...........Semblance.

Spider Ophrys................Adroitness. Skill.

Spiderwort...................Esteem, but not love. Transient love.

Spindle Tree................. Your image is engraved on my heart.

Spiræa......................Uselessness.

Spring Caroline ..............Disappointment.

Star of Bethlehem............Guidance.

St. John's Wort.............. You are a prophet. Superstition.

Stock, Ten Week.............Promptitude.

Stonecrop.....................Tranquillity.

Stramonium.................Disguise.

Strawberry..................Perfect excelcence.

Strawberry Tree.............Esteem and love.

Straw, Broken...............Rupture of a contract.

Sunflower, Dwarf............Adoration.

Sunflower, Tall...............Haughtiness.

Swallow-wort................Cure for heartacke. 
Sweet Brier..................Simplicity.

Sweet Brier, Yellow.........Decrease of love.

Sweet Flag ..................Fitness.

Sweet Sultan.................Felicity.

Sweet Sultan Flower......... Widowhood.

Sweetwilliam................Gallantry. Finessc. A smile.

Sycamore....................Curiosity.

Syringa.......................Memory .

Syringa, Carolina.............Disappointment.

Tamarisk.....................Crime.

Tansy........................I declare against you.

Tendrils of ClimbingPlants. Ties.

Thistle, Common.............Austerity. Emblem of Scotland.

Thistle, Scotch...............Retaliation.

Thorn, Black................. Difficulty.

Thorns, Branch of............Severity.

Thrift........................Sympathy.

Throatwort.................... Neglected beauty.

Thyme ........................Activity.

Tiger Flower.................For once may pridebefriend me.

Toothwort......................Secret love.

Touch-me-not ................. Impatient resolves.

Traveller's Joy ...............Safety.

Trefoil........................Revenge.

Tremella Nestoc..............Resistance. 
Truffle. Surprise.

Tuberose. Dangerous pleasures.

Tulip..........................Fame.

Tulip, Red.....................Declaration of love.

Tulip, Variegated.............Beautiful eyes.

Tulip, Yellow ...................Hopeless love.

Turnip ..........................Charity.

Valerian .......................Accommodating disposition.

Venus' Looking-glass .........Flattery.

Vernal Grass..................Poor, but happy.

Veronica ........................Fidelity.

Vervain ........................Enchantment.

Vetch...........................Shyness.

Vine.............................Drunkenness.

Violet, Blue ...................Faithfulness. Love.

Violet, Dame.................You are the queen of coquettes.

Violet, Purple.................. You occupy my thoughts.

Violet, White..................Innocence. Modesty.

Violet, Wild................... Love in idleness.

Virgin's Bower.................Filial love.

Wallflower....................Fidelity in misfortune.

Walnut.......................Intellect. Stratagem.

Wheat..........................Prosperity.

Whin ............................Anger.

Willow..........................Forsaken. 
Willow-Herb, Spiked.......Pretension.

Willow, Water...............Freedom.

Willow, Weeping.............Mourning.

Wolfsbane ..................... Misanthropy.

Woodroof......................Modest worth.

Wood Sorrel...................Joy. Maternal tenderness.

Wormwood...................Absence.

Yew..........................Sadness.

Zephyr Flower...............Expectation.

Zinnia....................... Thoughts of absent friends.

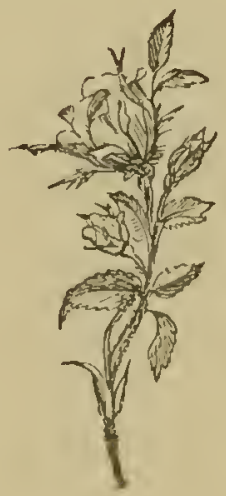




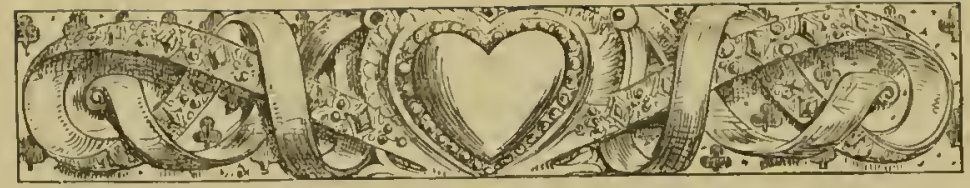

\section{THE LANGUAGE OF FLOWERS.}

\section{PART SECOND.}

Sentiments, and the dflowers which gifepresent them.

A bsence....................... Wormwood.

Abuse not....................Crocus.

Acknowledgment............Canterbury Bell.

Activity.....................Thyme.

Addresses, rejected........... Ice Plant.

Adoration..................... Dwarf Sunflower.

Adroitness....................Spider Ophrys.

Adversity, energy in.........Camomite.

Advice ........................Rhubarb.

Affection beyond the grave..Green Locust Tree.

Affection, bonds of...........Gilly Flower.

Affection, enduring........... Gorse.

Affliction .......................Aloe. 
Age..........................Snowball Tree.

Age, winter of...............Guelder Rose.

Agitation (quaking Grass. Moving Plant.

Ambition......................Mountain Laurel. Amiability...................White Jasmine. Am I forgotten ?...............Holly. Am I perfectly indifferent to you ?..................... Dogwood Blossom. Amusement...................Bladder Nut Tree. Anger........................ Whin.

Anticipation..................Gooseberry. Anxiety, tranquillize my...Christmas Rose. Anxious and trembling......Red Columbine. Ardour.........................Arum.

Argument...................Fig. Artifice .......................Acanthus. Assiduous to please..........Ivy Sprig, with tendrils. Attachment...................Ipomøa. Attachment, early...........Thomless Rose. Audacity.....................Larch. Austerity.....................Common Thistle. Avarice...................... Scarlet Auricula. Aversion .................... Indian Single Pink: Bantering.....................Southernwood. 
Pashfulness...................Peony.

Bashful shame...............Deep Red Rose.

Beautiful, call me not....... Unique Rose.

Beauty........................Party-coloured Daisy. Full Red Rose.

Beauty, a.................... Orchis.

Beauty always new..........China Rose.

Beauty and prosperity.......Red-leaved Rose.

Beauty, capricious...........Lady's Stipper. Mrush Rose.

Beauty, delicate.............Flower-of-an-hour. Hibiscus.

Venetian Mallow.

Beauty is your only attraction........................Japan Rose.

Beauty, magnificent......... Calla Ethiopica.

Beauty, mental...............Kennedya. Clematis.

Beauty, neglected............Throatwort.

Beauty, pensive.............Laburnum.

Beauty, rustic.................French Honeysuckle.

Beauty, transient.............Night-blooming Cereus.

Beauty, unconscious..........Burgundy Rose.

Beauty, unfading............Gilly Flower.

Belief........................Passion Flower.

Belle, a........................ Orchis.

Beneficence..................Marsh Mallow.

Benevolence.................Calycanthus. Potato. 
Betrayal.....................Judas Tree.

Betrayed..................... White Catchfly.

Beware........................Oleander. Rosebay.

Birth......................... Dittany of Crete.

Blackness.....................Ebony.

Bluntness ..................Borage.

Blushes.......................Marjoram.

Boaster, a...................Hydrangea.

Boldness .....................Larch. Pink.

Bonds........................Convolvutus.

Bound ......................Snowball.

Bravery and humanity...... Oak Leaf.

Brilliant complexion........Damask Rose.

Bulk..........................Gourd.

Bury me amid nature's

beauties...................Persimmon.

Business..................... Dodder of Thyme.

Busybody....................Quamoclit.

Calm .........................Buckbean.

Calumny......................Hellebore. Madder.

Care..........................Dodder of Thyme.

Change.......................Pimpernel.

Charity.....................Turnip.

Charming .................... Ifusk Rose Cluster.

Cheerful, always..............Coreopsis. 
Cheerfulness under misfortune.......................Chinese Chrysanthemum.

Childishness..................Buttercup.

Chivalry ......................Great Yellow Daffodil.

Cleanliness ..................IIyssop.

Cold-hearted..................Lettuce.

Colduess.......................Agnus Castus.

Comfort......................Pear Tree.

Comforting....................Scarlet Geranium.

Compassion...................Calycanthus. Elder.

Complacence..................Reed.

Concert.......................... Nettle Tree.

Coufidence....................IIepatica. Liverwort. Lilac Polyanthus.

Confidence in Heaven.......Flowering Reed.

Conjugal love.................Linden.

Consolation...................Snowdrop. Red Poppy.

Constancy....................Cedar Tree. Blue Hyacinth.

Bluebell.

Content.......................Houstonia.

Coquetry ..................... Day Lily.

Cordiality ....................Peppermint.

Counterfeit...................Mock Orange.

Courage.....................Black Poplar.

Crime.......................Tamarish. 
Criticism.....................Cucumber.

Cruelty.........................Nettle.

Cure..........................Balm of Gilead.

Cure for Heartache..........Cranberry. Pleurisy Root.

Swallow-wort.

Curiosity.....................Sycamore.

Danger......................Rhododendron.

Dangerous pleasures.........Tuberose.

Dark thoughts................Nightshade.

Dauntlessness ................Sea Lavender.

Death......................Cypress.

Deceit.......................Apocynum. Dogsbane.

Deceitful charms............Thorn Apple.

Deception.................... White Cherry Tree.

Defect ........................Henbane.

Dejection.....................Lichen.

Delay ........................Eupatorium. Fever Roots

Delicacy......................Cornbottle. Bluebottle.

Departure..................... Sweet Pea.

Despair....................... Cypress and Marigold.

Devotion......................Iteliotrope.

Difficulty.....................Black Thorn.

Diffidence.....................Sowbread.

Dignity.....................Cloves. Elm. [rolina.

Disappointment.............Spring Caroline. SyringaCa- 
Discretion......................temon Blossom. Maiden Hair.

Disdain........................ Yellow Carnation. Rue.

Disguise........................ Stramonium.

Disgust.........................Frog Ophrys.

Disposition, accommodating... Valerian.

Dissension.....................Pride of China.

Distinction....................Cardinal F'lower.

Distrust........................Lavender.

Docility........................Rush. Bulrush.

Do not abuse .................. Saffron Flower.

Domestic economy...............Itouseleek.

Domestic happiness.............Monthly Honeysuckle.

Domestic industry...............Flax.

Domestic virtues................Sage.

Dreams......................... Osmunda.

Drunkenmess.................... Vine.

Duration.........................Dogwood.

Eclat............................Indian Cress.

Education.......................Cherry Tree.

Egotism........................Poet's Narcissus.

Elegance.......................Pink Acacia. Locust Tree.

Elegance, mature ...............Pomegranate Flower.

Elevation........................Fir Tree.

Eloquence.......................Lagerstrcemia. Lotus. 
Embarrassment..............Love-in-a-puzzle.

Enchantment................Vervain. Holly Herb.

Encouragement..............Golden Rod.

Ennui........................Mosses.

Enthusiast, a religious......Lychnis.

Envy........................Crane's Bill. Bramble.

Error..........................Bee Ophrys.

Error, paternal..............Cardamine.

Esteem.......................Sage.

Esteem and love..............Strawberry Tree.

Esteem, but not love.........Spiderwort.

Excellence, perfect...........Strawberry.

Excellence, unpretending...Camellia Japonica.

Excess, beware of............Saffron Flower.

Expectation...................Zephyr Flower.

Expectation, disappointed..Fish Geranium.

Extent........................Gourd.

Extravagance, fantastic......Scurlet Poppy.

Eyes, beautiful...............Variegated Tulip.

Eyes, sun-beamed ............Scarlet Lychnis.

Facility.......................Germander Speedwell.

Faithfulness...................Blue Violet. Heliotrope.

Falsehood.....................Bugloss. Dogsbane. Manchineal Tree. Yellow Lity.

Fame........................ Tutip. 
Farewell ....................Spruce Pine.

Fascination..................Carnation. Honesty.

Fashion......................Lady's Mantle.

Fashionable...................Queen's Rocket.

Fate........................Ilemp.

Fate, the colour of my ......Coral IIoneysuchle.

Feasting......................Parsley.

Fecundity...................IIollyhock.

Felicity......................Centaury. Sweet Sultan.

Female ambition.............. White IIollyhoch.

Female fidelity...............Speedwell.

Female loveliness, the per-

fection of.... Justicia.

Fickleness....................Lady's Slipper. Pink Larkspur.

Fidelity.......................Ivy. Veronica.

Fidelity in love...............Lemon Blossoms.

Fidelity in misfortune...... Wallflower.

Filial love.................... Virgin's Bower.

Fine arts, the................Acanthus.

Finesse.......................Sweetwilliam.

Fire..........................IIorehound.

Fitness....................... Sweet Flag.

Flame........................Fleur-de-lis. Yellow Iris.

Flattery ..................... Venus' Looking-glass.

Flee away..................Pennyroyal. 
Folly ........................Columbine.

Foolishness..................Pomegranate.

Force........................Fennel.

Foresight.....................Holly.

Forgetfulness.................Moonwort.

Forget me not................Forget-me-not.

Forsaken....................Garden Anemone. Laburnum.

Willow.

Frankness.................... Osier.

Freedom .....................Water Willow.

Frieuds, thoughts of absent..Zinnia.

Friendship...................Rose Acacia. Ivy.

Friendship, early .............Red Periwinkle.

Friendship, unchanging.....Arbor Vitce.

Frivolity.....................Bladder Nut Tree. London Pride.

Frugality.....................Chicory. Endive.

Gaiety.......................Butterfly Orchis. Yellow Lily.

Gain.........................Cabbage.

Gallantry....................A nosegay. Sweetwilliam.

Generosity.................... Orange.

Genius.......................Plane Tree.

Gentility...................Geranium. Pompon Rose.

Gladness...................Myrrh. 
Gladness, youthful...........Spring Crocus.

Glory........................Bay Tree. Mountain Lau. rel.

Good-nature...................Mullen.

Goodness ..................... Bonus Henricus.

Gossip ........................Cobcea.

Grace and elegance...........Y Yellow Jasmine.

Grandeur.......................1sh T'ree.

Gratitude...................... White Bell Flower.

Grief ..........................Aloe. Harebell. Marigold.

Guidance.....................Star of Bethlehem.

Happiness .....................Mugwort.

Happiness, return of.........Lily of the Valley.

Happy love....................Bvidal Rose.

Hatred.........................Sweet Basit.

Haughtiness..................Double Larkspur. Tall Sunflower.

Health ......................... Iceland Moss.

Heart, the incense of a faitl-

ful..........................Frankincense.

Heart's mystery, the........Crimson Polyanthus.

Heartlessness................. Hydrangea.

Hermitage.....................Ifilkwort.

Honesty.....................Ilonesty.

Hope........................IIawthorn. Snowdrop. 
Hope, extinguished...........Major Convolvulus.

Hope in adversity............Spruce Pine.

Hopeless love................. Yellow Tutip.

Hopeless, not heartless......Love-lies-bleeding.

Horror........................Mandrake. Dragonwort.

Snakesfoot.

Hospitality..................Oak Tree.

Humility.....................Broom. Field Lilac. Small

Bindweed.

Hypocrisy....................Manchineal Tree.

I am dazzled by your charms. Ranunculus.

I am worthy of you......... Full White Rose.

I am your captive............Peach Blossom.

I aspire to thy smile......... Daily Rose.

I attach myself to you........ Indian Jasmine.

I change but in death .......Bay Leaf.

I declare against you........Belvedere. Tansy. Wild $\mathrm{Li}_{i}$ quorice.

I desire to please.............Mezerion.

I desire a return of affection. Jonquit.

I die if neglected............. Laurestina.

I engage you for the next dance.......................Ivy Geranium.

I feel all my obligations..... Lint.

I feel your kindness.........Flax. 
I have a message for you.........................tris.

I have lost all................ Mourning Bride.

I live for thee.................Cedar Leaf.

I love........................Red Chrysanthemum.

I partake your sentiments..Double China Aster.

I shall die to-morrow.........Gum Cistus.

I share your sentiments.....Garden Daisy.

I will not answer hastily...Monthly Honeysuckle.

I will not survive you.......Black Mulberry.

I will think of it.............Single China Aster. Wild Daisy.

I wish I were rich............Kingcup.

I wound to heal..............Eglantine.

Idleness.......................Mesembryanthemum.

If you love me, you will discover it....................Maiden-blush Rose.

Ill-temper....................Barbervy.

Immortality................Amaranth.

Impatience ..................Balsam.

Importunity.................Burdock. Fuller's Teasel.

Inconstancy..................Evening Primrose.

Inconstancy in love.......... Wild Moneysuckle.

Incorruptible ................. Cedar of Lebanon.

Independence............... Wild Plum Tree. 
Indifference..................Senvy. Candytuft. Agnus Castus. Mustard Seed.

Indiscretion....................Split Reeds. Almond Tree. Industry.......................Bee Orchis. Red Clover:

Ingeniousness................ White Pink.

Ingenuity ....................Pencit-leaved Geranium.

Ingenuous simplicity.........Mouse-eared Chickweed.

Ingratitude ...................Buttercup. Wild Ranunculus.

Injustice ....................Hop.

Innocence.................... White Daisy. White Violet.

Innocence, youthful.......... White Lilac.

Insincerity...................Foxglove.

Insinuation...................Great Bindweed.

Inspiration ...................Angelica.

Instability...................Dahlia.

Intellect....................Walnut.

Ireland, emblem of.......... Shamrock.

Jealousy .....................French Marigold. Yellow Rose.

Jest .............................Southernwood.

Joy ...........................Wood Sorrel.

Joys to come................. Celandine.

Juice..........................Fir of Gilead.

Justice.........................Rudbeckia.

Justice, do me...............Chestnut Tree.

Justice shall be done.........Coltsfoot. 
Knowledge, useful...........Parsley.

Lady, deign to smile......... Oak Geranium.

Lamentation...................Aspen Tree.

Levity ........................Larkspur.

Life...........................Lucerne.

Light-heartedness ............Shamrock.

Lightness.....................Larkspur.

Longevity....................Fig.

Love..........................Blue Violet. Myrtle. Rose.

Red Bay.

Love, a heart ignorant of... White Rosebud.

Love, ambassador of.........Rose Cabbage.

Love, ardent.................Balsam.

Love at first sight...........Arkansa Coreopsis.

Love, bond of................. Mronthly Honeysuckle.

Love, chaste..................Acacia.

Love, confession of............Moss Rosebud.

Love, consumed by...........Syrian Mallow.

Love, declaration of..........Red Tulip.

Love, decrease of.............Y Yellow Sweetbrier: Yellow Rose.

Love, estranged..............Lotus Flower.

Love in idleness .............. Wild Violet.

Love is dangerous...........Carolina Rose.

Love, only deserve my......Campion Rose.

Love, platonic................Rose Acacia. 
Love, pretended..............Catchfly.

Love, pure and ardent......Red Double Pink.

Love returned................Ambrosia.

Love, secret...................Toothwort. Yellow Acacia.

Love, slighted.................Yellow Chrysanthemum.

Love, sweet and secret...... Honey Flower.

Love, the first emotions of.. Purple Lilac.

Love, true ...................Forget-me-not.

Lovely, always................ Indian Double Pink.

Lovely, thou art all that is..Austrian Rose.

Love's oracle..................Dandelion.

Lowliness.....................Bramble.

Luxury .....................Chestnut.

Majesty......................Crown Imperial. Imperial Lily.

Malevolence..................Lobelia.

Marriage.....................Saffron.

Maternal affection............ Cinquefoil.

Maternal love.................Mross.

Maternal tenderness......... Wood Sorrel.

Matrimony...................Ivy.

Mcanness.....................Cuscuta. Dodder.

Meekness.....................Birch Tree.

Mecting, an appointed......Everlasting $P$ ea.

Mecting, an expected ........Nutmeg Geranium. 
Melancholy Dark Geranium. Cypress and Marigold.

Melancholy mind............Sorrowful Geranium.

Memory......................Red Bay. Sypinga.

Memory, pleasures of........Btue Periwinkle.

Merit, concealed.............Coriander.

Merit, reward of .............Bay Wreath.

Merit, superior..............Full Moss Rose.

Merit, unpatronized..........Red Primrose.

Mirth.......................Saffron Crocus.

Misanthropy.................Fuller's Teasel. Wolfsbane.

Modest worth.................Woodroof.

Modesty......................White Lilac. White Violet.

Mourning.....................Cypress. Weeping Willow.

Music........................ Oats.

My bane, my antidote...... White Poppy.

My best days are past.......Meadow Saffron.

My eompliments.............Iris.

My regrets follow you to

the grave....................Asphodel.

Nature, love of ...............Magnolia.

Neatness .....................Broom.

Night........................Minor Convolvulus. Night

Convolvulus.

Obstaele.......................liest IIarrow. 
56 TIIE LANGUAGE OF FLOWERS.

Obstacles, I surmount all...Mristletoe.

Ornament....................IIornbeam Tree.

Painting .....................A uricula.

Painting the lily ..............Daphne Odora.

Parental affection.............Sorrel.

Participation................. Double Daisy.

Passion ...................... White Dittany. Yellow Iris.

Paternal error................Cardamine.

Patience.....................Dock. Ox Eye.

Patriotism...................Nasturtium.

Peace.......................... Olive Branch.

Pensiveness..................Cowstip.

Perfection....................Pine Apple.

Perfidy.......................Common Laurel.

Perform your promise......Plum Tree.

Perplexity....................Love-in-a-mist.

Persecution ...................Chequered Fritillary.

Perseverance.................Canary Grass. Swamp Mragnolia.

Persuasion....................Althaca Frutex.

Philosophy.................Pitch Pine.

Piety, steadfast.............. Wild Geranium.

Pity..........................Black Pine.

Plan ........................... Nettle Tree.

Play.........................IIyacinth. 
Pleasant recollections........ White Periwinkle.

Pleasantry...................Gentle Balm.

Pleasure and pain............Dog Rose.

Pleasure, evanescent........Poppy.

Pleasure, lasting............. Everlasting Pea.

Poetry.......................Eglantine.

Poor, but happy.............. Vernal Grass.

Poverty........................Evergreen Clematis.

Precaution.....................Golden Rod.

Precocity......................Ifay Rose.

Preference...................Apple Blossom. Rose Geranium.

Preference, present..........Apple Geranium.

Presumption..................Snapdragon.

Pretension....................Lythrum. Willow-herb, Spiked.

Prettiness....................Pomme Rose, or Rose-Apple.

Pride..........................Amaryllis. Ilundred-leaved Rose.

Pride befriend me, for once may.......................Tiger Flower.

Pride of riches................ Polyanthus.

Privation.....................Indian Plum. Myrobalan.

Profit........................Cabbage.

Profuseness..................Fig Tree.

Prohibition...................Privet. 
58 THE LANGUAGE OF FLOWERS.

Promptitude................Ten Week Stock.

Prosperity...................Beech Tree. Wheat.

Protection.....................Bearded Crepis. Juniper.

Prudence......................Mountain Ash. Service Tree.

Purity ....................... White Lilac.

Purity and sweetness........ White Lily.

Quick-sightedness ............ Hawkweed.

Reason.........................Goat's Rue.

Recall.........................Silver-leaved Geranium.

Recantation....................Lotus Leaf.

Reconciliation.................Filbert. Hazel.

Refusal.......................Striped Carnation. Variegated Pink.

Regard......................Daffodil.

Relief........................Balm of Gilead.

Religious superstition.......Passion Flower.

Remembrance................Rosemary. Forget-me-not.

Remembrance, never-ceasing.........................Cudweed.

Remembrances, sorrowful...Adonis.

Remorse......................tramble. Raspberry.

Rendezvous...................Chickweed.

Repose.......................Buckbean.

Reserve......................Maple.

Resistance...................Tresnella Nestoc. 
Resolution...................Purple Columbine.

Resolves, impatient.........Touch-me-not.

Restoration ..................Persicaria.

Retaliation..................Scotch Thistle.

Revenge......................Trefoil.

Reverie ......................Flowering Fern.

Riches .......................Corn.

Rigour.......................Lantana.

Rivalry ......................Rocket.

Royalty......................Angrec.

Rudeness.....................Clotbur.

Rupture of a contract........Broken Straw.

Sadness.......................Dead Leaves. Yew.

Safety.........................Traveller's Joy.

Satire .........................Prickly Pear.

Scandal.......................Hellebore.

Scepticism ....................Nightshade.

Scotland, emblem of .........Common Thistle.

Sculpture......................Iloya.

Secrecy........................Full-blown Rose placed over two buds.

Self-esteem ....................Poet's Narcissus.

Selfishness....................Dodder of Thyme.

Semblance....................Spiked Speedwell.

Sensitiveness..................Mimos 
Sensuality...................Spanish Jasmine.

Sentiment, warmth of.......Spearmint.

Separation...................Carolina Jasmine.

Serenade, a................. Dew Plant.

Severity.....................Branch of Thorns.

Shame......................Peony.

Sharpness...................Barberry.

Shyness...................... Vetch.

Sickness.....................Field Anemone.

Silence .........................Belladonna.

Simplicity................... Sweet Brier.

Simplicity, ingenuous........Blaeberry. Mouse-eared Chickweed.

Sincerity.....................Fern. Honesty. Satin Flower. Skill ..........................Spider Ophrys.

Slander........................Snake's Lounge. Burning Nettle.

Sleep .......................... White Poppy.

Smile, a.......................Sweetwilliam.

Snare.........................Catchfly. Dragon Plant.

Solitude......................Heath. Lichen.

Sorcery .......................Nightshade.

Sorrow.......................Purple Hyacinth.

Sourness .....................Barberry.

Spleen......................Fumitory. 
Splendour................... Scarlet Nasturtium.

Sport........................Hyacinth.

Stability

Cresses.

Steadfast piety Wild Geranium.

Stoicism Box.

Stratagem....................Walnut.

Strength ....................Fennel. Cedar Tree.

Stupidity.....................Scarlet Geranium. Almond

Tree.

Submission...................Grass. Harebell.

Success crown your wishes.. Coronilla.

Succour......................Juniper.

Superstition ..................St. John's Wort.

Surprise.....................Betony. Truffle.

Susceptibility................Passion Flower.

Suspicion....................Champignon.

Sympathy...................Balm. Thrift.

Talent ........................ White Pink.

Taste .........................Scarlet Fuchsia.

Tears............................Helenium.

Temperance...................Azalea.

Temptation...................Apple. Quince.

Thankfulness .................Agrimony.

Ties...........................Tendrits of climbing plants.

Time.........................Pitch Pine. White Poplar. 
Timidity ....................Amaryllis. Marvel of Peru.

Token, a..................... Ox-Eye Daisy. Laurestina.

Touch me not................Burdock.

Tranquillity..................Maidwort. Stonecrop.

Tranquillize my anxiety....Christmas Rose.

Transient impression........ Withered White Rose.

Transient love................Spiderwort.

Transport of joy..............Cape Jasmine.

Treachery.....................Bilberry.

Truth....................... White Chrysanthemum. Bitter.

Night Shade.

Unanimity..................Phlox.

Unbelief .....................Judas Tree.

Uncertainty ..................Convolvulus.

Unchangeable................Globe Amaranth.

Unconscious.................Red Daisy.

Unfortunate attachment....Mourning Bride.

Unfortunate love.............Scabious.

Union........................tancaster Rose.

Unity........................ White and Red Rose.

Uselessness...................Diosma. Spircea. Meadowsweet.

Utility.......................Grass.

Variety.....................China Aster. Mundi.

Vice.........................Darnel. Ray Grass.

Victory ......................Palm. 
Virtue........................Mint.

Virtue, reward of............Crown made of Roses.

Vivacity......................Houseleek.

Voraciousness .................Lupine.

Vulgar-minded..............African Marigold.

War...........................Milfoit. York Rose.

Warlike trophy...............Indian Cress. Nasturtium.

Warmth.....................Peppermint.

Weakness....................Moschatel. Crowfoot Mush:

Widowhood....................Sweet Sultan Flower. Sweet Scabious.

Wiuning grace...............Cowstip.

Wisdom.......................Mulberry Tree.

Wish, a......................Foxglove.

Wit...........................Meadow Lychnis. Ragged Robin.

Wit, ill-timed ...............Wild Sorrel.

Witchcraft.....................Nightshade.

Woman's love................Carnation. Carnation Pink.

Worth, modest............... Woodroof.

You are a prophet............St. John's Wort.

You are aspiring.............Mountain Pink.

You are cold .................IIortensia.

You are hard................Ebony.

You are merry...............Mundi Rose. 
You are my divinity.........American Cowslip.

You are rich in attractions.. Garden Ranunculus.

You are the queen of coquettes.......................Queen's Rocket. Dame Violet.

You are young and beautiful.Red Rosebud.

You have no claims.........Pasque Flower.

You make no pretension....Flora's Bell.

You occupy my thoughts...Pansy. Purple Violet.

You please all.................Currants.

You will be my death.......Hemlock.

Your image is engraved on my heart.....................Spindle Tree.

Your looks freeze me.........Ficoides.

Your presence revives me...Rosemary.

Your presence softens my pain ...........................Aillkvetch.

Your purity equals your loveliness...................... Orange Blossom.

Your qualities surpass your charms. .Mignonnette.

Youth, early ..................Primrose.

Youthful love..................Red Catchfy.

Zealousuess .....................Elder.

Zest. Lemon. 
Whaten

\section{THE POETRY OF FLOWERS.}

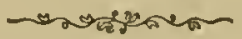





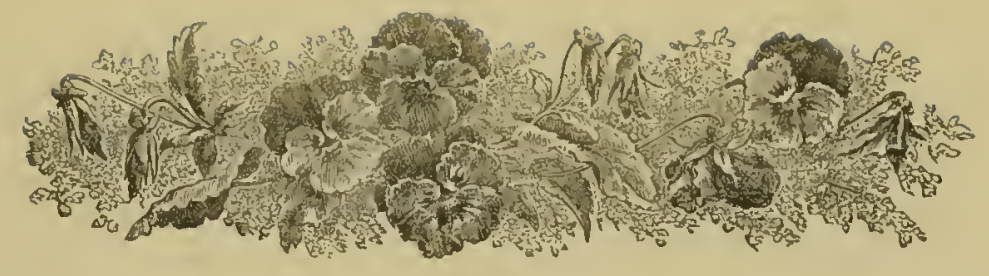

\section{THE POETRY OF FLOWERS.}

\section{THE USE OF FLOWERS.}

203 OD might have made the earth bring forth Enough for great and small, The oak-tree and the cedar-tree, Without a flower at all.

$\therefore$ We might have had enough, enough For every want of ours, For lux'ry, medicine, and toil, And yet have had no flowers.

The ore within the mountain mine Requireth none to grow ; Nor doth it need the lotus-flower T'o make the river flow. The clouds might give abundant rain, The nightly dews might fall, 
And the herb that keepeth life in man

Might yet have drunk them all.

Then wherefore, wherefore were they made, All dyed with rainbow light,

All fashioned with supremest grace,

Upspringing day and night,-

Springing in valleys green and low,

And on the mountains high,

And in the silent wilderness,

Where no man passes by?

Our outward life requires them not-

Then wherefore had they birth ?

To minister delight to man,

To beautify the earth;

To comfort man, to whisper hope,

Whene'er liis faith is dim,-

For who so careth for the flowers

Will much more care for him.

MARY HOWITT. 


\section{ACACIA, YELLOW.}

(Secret Love.)

Thou, like a star-flower in the wood, Thy modest charms art hiding; Content with humbly doing good, And in God's love abiding.

But though the world observes thee not, In one fond heart thou'rt treasured; And bright indeed must be the lot That shares a love unmeasured.

\section{A M A R A N T H.* \\ (Immortality.)}

OH! not for the hue of thy roseate cheek, Nor the dimpled rubies that smile and speak;

Oh! not for the flash of thy glowing eye, Nor the eloquent sound of thy soft, low sigh, Do I love thee, bright being of passion and grace. 'Tis the soul, the sweet soul in thy beautiful face, The spirit immortal, the charm that ne'er dies, That from deatl and the gloom of the grave will arise;

* Of this plant there are many species. The prettiest is the Globe Amaranth-flowers red, unfading. 
It is this that enthrals me: and thou unto me Art the embryo only, of what thou shalt be; For thy mortal shall die, but the beauty I love Hath an endless existence and progress above!

\section{BALSAM.}

(Impatience.)

I CANNoT, will not longer brook

Thy cold delay, thy prudent look.

Dost love me? Share at once my fate,

Be it or bright or desolate.

I will abide no half-way love,

Nor wait for prudence ere I move ;

One more repulse, and I depart!

Come now, or never, to my heart.

\section{BLUE BELL.}

(Constancy.)

They bid me forget him! as if I could tear

From my heart the dear image so long cherished there;

Like a rose in the wilderness, blooming and free,

Like a rose in the desert, that love is to me. 
I brood o'er my thoughts in the stillness of night;

I cannot forget him-would not, if I might!

'Tis the star that illumines my desolate way, And gives it the glory and brightness of day.

\section{CAMOMILE.*}

(Energy in Adversity.)

ONWARD! Hath earth's ceaseless change

Trampled on thy heart?

Faint not, for that restless range

Soon will heal the smart.

Trust the future-time will prove

Earth hath stronger, truer love.

* * * * *

Bless thy God, the heart is not

An abandoned urn,

Where, all lonely and forgot,

Dust and ashes mourn;

Bless Him, that His mercy brings

Joy from out its withered things.

* This plant has a white or yellow flower, and is much loved for its fragrance. 


\section{CANTERBURY BELL。}

(Acknowledgment.)

AH, must I tell thee? Well, I fear

The die is surely cast

That I am thine, and only thine,

Beloved, to the last.

I could not see thee, hear thy voice,

Or look upon thy brow,

Nor fail to love thee tenderly-

My heart must break or bow.

\section{CARNATION, YELLOW.*}

(Disdain.)

IT is not well amid thy race to move

And shut thy heart to sympathy and love;

It is not well to scorn inferior minds,

And pass them by as though they were but hinds.

Pride may become thee, as the veil a nun;

But ah! they love thee not whom thou dost shun!

And days may come to thee when human love

Thou wilt desire all earthly things above;

* This is a very rich and fragrant flower, growing sometimes as large as a rose. 
And thou wilt mourn that, in thy days of pride, Thou didst not win some true hearts to thy side; Wilt mourn that, now thy rank and wealth have flown, Thou'rt left to suffer and to die-alone.

\section{CATCHFLY, RED.}

(Youthful Love.)

Never forget the holy love

It liath been ours to keep

Undimmed amid all cares and toils-

The true, the pure, the deep.

The trusting love of early youth,

Still fair in its own changeless truth.

Never forget-it hath been joy,

In suffering and in tears,

To know that thou wert still the same

As in our earlier years.

The cup of life were bitterer yet,

Could I but deem thou wouldst forget. 


\section{CHINA ASTER, DOUBLE.*}

(I Partake your Sentiments.)

YeS, I am thine! Upon thy bosom leaning, No grief hath power to damp my fervent bliss;

Nor can such love to thee be overweening--

Thou art deserving all, and more than this!

Beloved, ne'er from thee one moment straying, My heart shall twine its roots upon thy truth; All lighter loves than this are fast decaying, Lost in the dying years of sunny youth.

\section{CLEMATIS.† \\ (Mental Beauty.)}

Excelling riches dwell within thy mindStrong, fervent thought, and eloquence refined;

Ideal beauty clusters round thy soul, While deep within the waves of feeling roll. Religious fervour mingles with the grace Of playful fancy to illume thy face,

* The Aster is a very extensive genus, but there is none so beautiful as the cultivated China Aster.

$\dagger$ This is a very graceful climbing-plant, bearing white and pale-blue flowers. 
And sparkling wit with graver sense unites, And fills thine cye with many changing lights.

Oh! beautiful indeed, a mind like thine!

And well might angels bow at such a shrine.

But man, weak man, oft passes idly by,

To worship beauty that attracts the eye ;

While mental grace, a charm that ne'er can fade,

Flies from the crowd, and dwells amid the shade.

\section{COWSLIP.* \\ (Winning Grace.)}

Sue grew in love. Around her infant home Life hung its summer hues, and very fair Was this wild earth to her. She learned to roam

In artless radiance where the woodland air Showered trembling sweetness on the glancing streams, And stole its hue from sunset's golden beams.

She twined the orchis in her hazel bair, And stole the violets from the brook-side dell:

The wilding race was her peculiar care;

Her dearest music was the foxglove's bell, When the wild bce with his transparent wings Stirs the sweet air, and makes believe he sings.

* A very bright, beautiful yellow flower. 


\section{DAHLIA.*}

(Dignity.)

IT is worth much, in this dull world of strife And foolish vanity, to meet a heart Serene and beautiful like thine! The praise And selfish flattery of the heartless crowd Fall idly on thine ear, whilst thou unmoved, And with a lofty purpose in thy breast, Retain'st thy elevation o'er the herd, No less by that calm majesty of soul Which shrinks from adulation, than by gifts Of lofty intellect and outward grace. Thy form hath elegance that indicates The beautiful refinement of thy thoughts; And there is dignity in thy firm step, That speaks a soul superior to the thrall Of petty vanity and low-born pride.

* This is a Mexican plant, much cultivated by modern horticulturists. The flowers are of all colours, and very showy; growing often to the height of six or eight feet. 


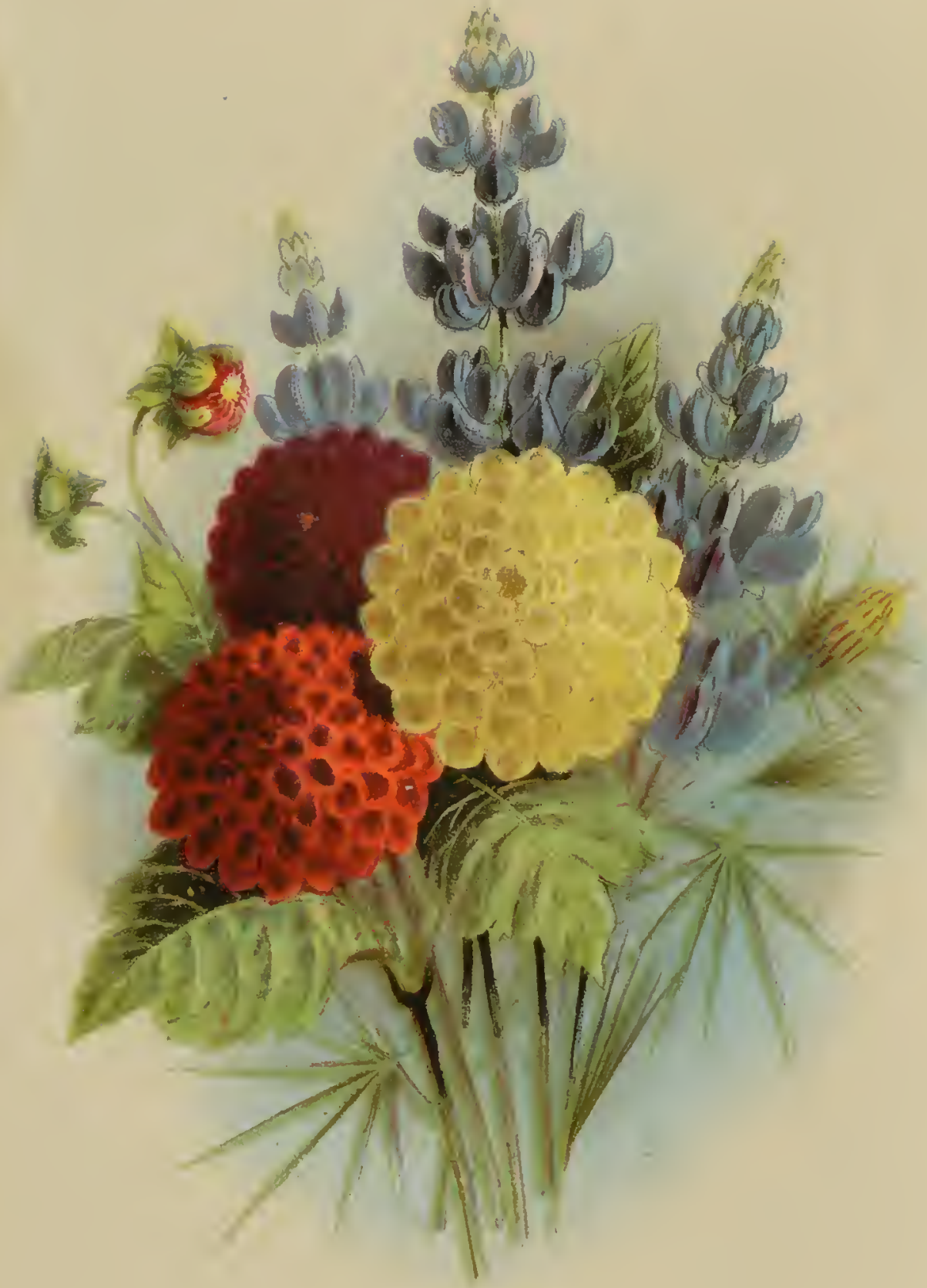





\section{EGLANTINE.* \\ (Poetry.)}

THY spirit has a gift, a secret gift,

Which answers only to the far, bright stars,

When through the greenwood's high and changeful rift

Streams down the light of Venus and of Mars;

Which answers only to the winds and streams,

The sweet wood-blossoms and the moon's pale beams.

Thou seest strange beauty in the silent things

That others idly pass. The small, wild bird,

That flutters o'er the rose his bright blue wings;

The singing brook, by careless ears unheard;

The wild flower, swinging in the lonely dell,--

All bind thee with a strong and wondrous spell.

\section{FORGET-ME-NOT.†}

(True Love.)

Give not to weary thought the love That should be solely mine;

Nor tire thee of the shrinking dove

Thou call'st so fondly thine.

* This is a sweet-scentcd shrub, with delicate pink flowers. Grows often to a great height.

$\dagger$ A very pretty, but minute flower, growing by the brook-sides. Colour, sky-blue. 
I know my words are weak and smallFor mind like thine unmeet; But I have love beyond them all, To lavish at thy feet.

Nay, heed it not, this foolish tear That trembles in mine eyc;

It always comes when thou art here,-I know not how or why.

It is not grief, or pain, or joy,It comes of love, maybe;

Then do not thou the spell destroy'Tis bliss to weep for thee!

\section{GENTIAN.* \\ (Virgin Pride.)}

Alone and pure my life shall be, A vestal flame from passion free; Unwon, unwooed by human love, My heart shall fix its hopes above.

Approach me not with tempting wilesMy lip denies thee while it smiles;

In virgin liberty and peace

I will live out my mortal lease.

* A very beantiful autumn wild flower, of a deep blue colour, with delicately fringed petals. 


\section{GERANIUM, SCENTED.*}

(Preference.)

OTHERs may wear a gayer smile,

And speak in richer tones;

But ah! my heart, my heart, the while

Each spell save thine disowns.

Dearer to me one word of thine

Than all that others speak;

My heart I lay upon thy shrine-

Accept it ere it break.

\section{GILLY FLOWER.†}

(Unfading Beauty.)

O faint indeed are outward lues,

Compared with thy rich mental light;

Each day thy thoughts their rays diffuse,

Yet grow each added dily more bright.

To scatter charms so rich as thine,

In vain Time's surging billows roll;

* Leaves rose-scented. Flowers purplish-pink.

$\dagger$ Flowers bright red, purple, or white. The white Gilly Flower is very fragrant. 
The pearls that on thy forehead shine Are gathered daily from thy soul.

\section{HAWTHORN.*}

(Hope.)

Hope on, hope ever!

Dark o'er us now the clouds of grief are brooding, Hoarsely the streamlets murmur at our feet; Bright birds of song, our eager grasp eluding,

Far from our tree of love and life retreat. But oh! not yet, my gentle friend, shall leave us The fervent hope of sunshine and of joy; And whatsoe'er of wrong may come to grieve us, Let there be one thing grief can ne'er destroyHope on, hope ever!

\section{HONEYSUCKLE, WILD.†}

(Devoted Love.)

I Go with thee! I will be thine In weal, in want, in woe; Thy path, where'er it learls, is mineI go, my love, I go!

* Flowers white. Berries scarlot.

† Flowers white, red, scarlet. 
THE POETRY OF FLOWERS.

"Tis not for wealth I seek the shade

Of forest bower and tree;

To share the burdens on thee laid-

For this I go with thee.

\section{HYDRANGEA.*}

(Heartlessness.)

YES, thou canst smile and be as gay

As though no heart thy guile had broken;

While every step along my way

Brings up of thee some painful token.

Thou breathest in a dozen ears

The same fond words once breathed to me;

While I, alas ! in secret tears,

Can only think and dream of thee.

\section{IRIS.†}

(I have a Message for Thee.)

WERE not thy spirit purified to look

Through all things beautiful to God and heaven, These gentle readings from love's holy book

Had not been given.

* Flowers rose colour, sometimes blue. IIue very ehangeable.

$\dagger$ Flowers of various eolours-commonly blue. (499) 
Were thine eye sealed to those sweet lessons, taught In the dim oracles of leaf and tree,

I had not made them messengers of thought, Dear friend, to thee.

But take them now, for they will talk to thee In the sweet accents of poetic lore; Heed their soft pleadings-kindly "think of me"I ask no more.

\section{JASMINE, WHITE.* \\ (Amiability.)}

Thine is that excelling virtue The pure-hearted only know ; Thine that unassuming goodness Which in silent deeds doth flow.

Thou dost make the poor and needy In thy presence to rejoice; All the bowed and broken-liearted Love thy peace-inspiring voice.

- An Akiatic genus. Flowers white and verg fragrant. Plant climblng.

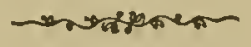




\section{JASMINE, YELLOW. \\ (Grace and Elegance.)}

LiKE the foam on the wave floating down to the sea, Like the zephyr that flits o'er the graiu-covered lea, Like the soft fleecy cloud o'er the face of the moon, Like the sail of a bird ou the still air of noon,So graceful, and airy, and gentle art thou, With thy curls floating free o'er thy radiant brow! So fairy-like moveth thy foot o'er the flowers, They look up and exclaim-" "Twas the step of the Hours!"

\section{JONQUIL.*}

(Affection Returned.)

'Тнат thou art loved, this flower my witness be !

In the bright moruing, noon, or starry night, One thought my bosom fills-it is of thee!

Aud thou dost make all hours and seasons bright.

To see thee, hear thee, know that thou art nigh,Oh, this is joy unkuown to me before! All other thoughts are gone when thou art by ; Thou fill'st my heart-it can contain no more!

* Flowers golden, emitting a pleasant but powerful perfume. 


\section{LARKSPUR, PINK.*}

(Fickleness.)

Tirou art not what thou wert,-

Farewell, and may God bless thee;

My heart with strength is girt

Once more to say, God bless thee!

Thou hast forgot thy vow-

I give thee back its token;

'Tis but a memory now

Of pledges lightly broken.

Farewell! we meet no more;

And though I now regret thee,

My grief will soon be o'er; -

I can, and will, forget thee.

\section{LILAC, PURPLE.†}

(First Emotion of Love.)

How sweet and rapturous 'tis to feel

Ourselves exalted in a lovely soul !-

To know our joys make glow another's cheek,

* Flowers blue, white, and pink. A very handsome, showy blossom, easy of cultivation.

$\dagger$ Too well known to need description. Flowers purple and white. 
Our fear's do tremble in another's heart, Our sufferings bedew another's eye !
As the little flowerct lideth
By the woodland stream,
So in youthful hearts abidetl
Love's first witching dream.

\section{LILY, WHITE. \\ (Purity and Sweetness.)}

Ask me not why I should love her:

Look upon those soul-full eyes!

Look while mirth or feeling move her,

And see there how sweetly rise

Thoughts gay and gentle from a breast

Whieh is of innocence the nest-

Which, though each joy were from it fled,

By truth would still be tenanted!

\section{LONDON PRIDE.}

(Frivolity.)

Love should have higher, nobler aims

Than mirth, and song, and dance;

Oh, then, from sport and idlc games

To higher dceds advance. 
Throw by thy foolish wit and songs, Thy graceful tricks of art; And, far from fashion's heartless throngs, Add wisdom to thy heart.

\section{MIGNONETTE.*}

(Your Qualities surpass your Charms.)

Beauty consists not in the sparkling eye, The damask cheek and lip, or forehead high ; Not in the graceful form, or glistening hair, Or melody of voice! Oh no! not there;But in the soul, which every glance displays Basking for ever in affection's raysSpeaking in love's soft tones, with sunlit smile, Which can an aching heart from woe beguile! It dwelleth there in majesty supreme, . Sweeter than music's voice, or seraph's dream!

\section{PANSY.† \\ (You occupy my Thoughts.)}

I HAVE sweet thoughts of thee!

They come around me like the voice of song; They come like birds that to the south belong,

* Flowers very fragrant. Colour greenisli-white, with yellow stamens. $\dagger$ This flower has three colours-purple, yellow, and blue. It is much cultivated, and highly esteemed. 

And wear a gayer wing and brighter crest Than those that on the roof-tree build the nest; They come more tender, beautiful, and bright Than any thoughts that others can excite;
They tell me gentle tales of thee and thine,- Of gems of truth that in thy spirit shine; Of goodness, purity, and holy zeal, That can for otliers earnest pity feel ; Of all things beautiful in soul and heart,- And such, they tell me, dearest, that thou art!

\section{PASSION FLOWER.}

(Religious Fervour.)

How should the soul with adoration glow,

To that great Power, eternal and supreme, Who gives us faculties for joy and woe,

And hope and reason guarding each extreme!

Who paints on sorrow's clouds the rainbow beam

That cheers our spirits through sad mists of tears, And bids the heaven-lit taper brighter gleam

As down the dark declivity of years We seek the better clime, where Truth her temple rears! 


\section{PEA, EVERLASTING.*}

(Wilt Thou go away?)

ONE moment o'er my chequered path Thy snile hath shed its gladdening ray;

A rainbow on a cloud of wrathAnd wilt thou, also, go away? * * * * * * Thou'rt going! Well, thou knowest What prayers arise for thee; And wheresoe'er thou goest, Bear gentle thoughts of me.

\section{PEA, SWEET.†}

(Departure.)

UnNoticed fell the sere and yellow leaf, Unheeded swept the morning breezes by; The fading flowers awoke no throb of grief, There was no sadness in the wind's low sigh; Could gloom or sorrow cloud the dying year, When thou, the summer of my heart, wert near?

One hour hath passed-and o'er the deep blue sky A dimness hangs, whose chill is in my heart;

* Flowers purple, crimson, and white.

$\uparrow$ Very beautiful, and possessing much of the fragrance of the Pink. The flowers are variegatcd with blue, lilac, rose, white, \&c. 


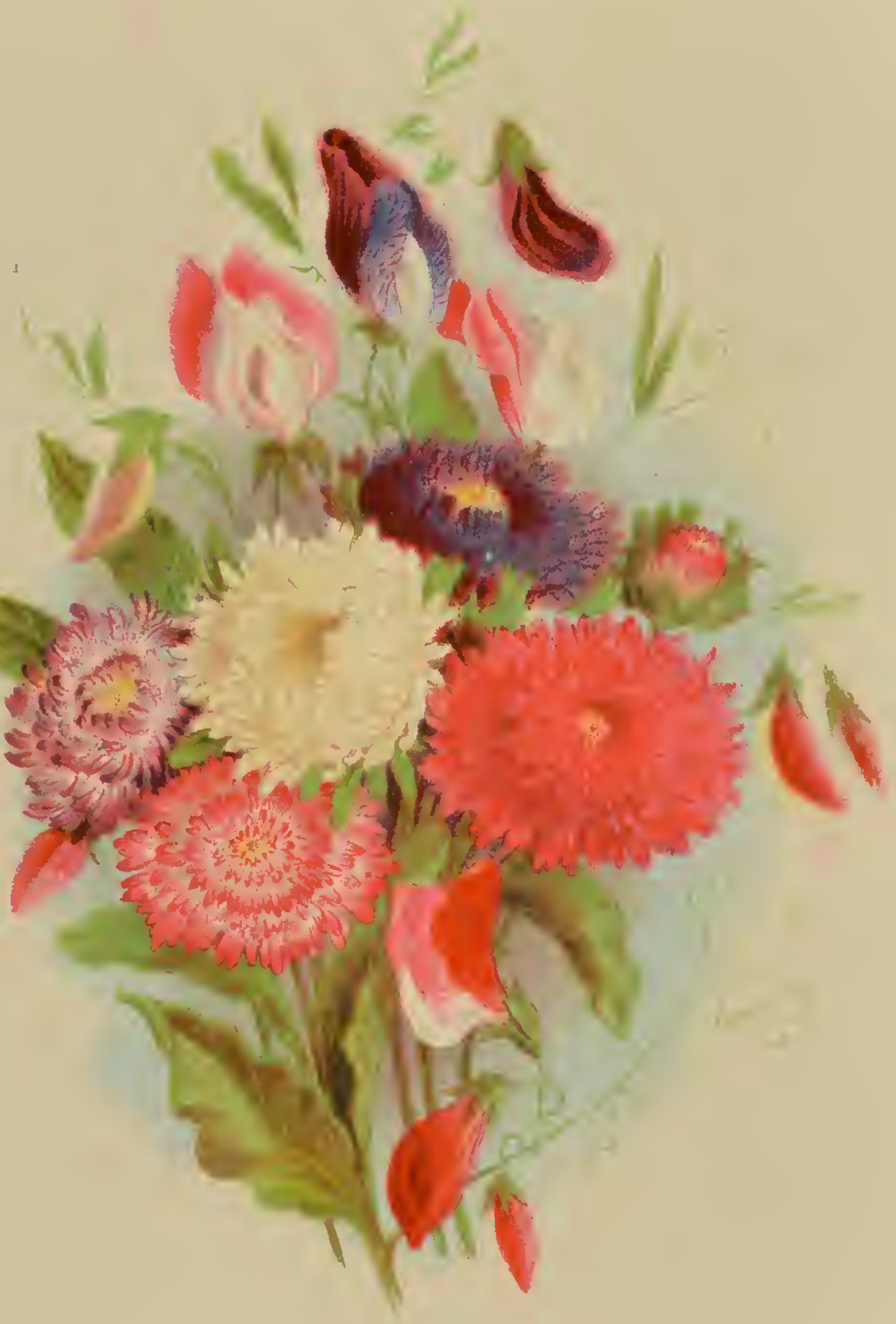



The wind with funeral moans goes sweeping by,

And asks in every whisper where thou art; The sunshine hath gone with thee and the flowers, And frost hath chained the fairy-footed hours.

\section{PEACH BLOSSOM.*}

(I am your Captive.)

OII, is it $\sin$ to love the very air

That once hath rested on thy beaming brow?

To gaze in fondness on thy vacant chair,

And on thy books and flowers, deserted now?

Or turn in worship on that pictured face,

Whose sweetest looks the heart alone can trace?

Is it a sin to live again each hour

Passed in thy presence?-to recall thy tones, Thy playful words, thy scrious thoughts, whose power Thrills every nerve my quickened spirit owns?

Is it a crime to worship and adore What is so good? - the Ideal asks no more.

\section{PHLOX.}

\section{(Unanimity.)}

Where'er thou goest, I will go ;

Where'er thou diest, die;

* Flowers beantiful, rose or pink colour. 
Together in one humble grave Our slumbering dust shall lie.

And I will love thy chosen friendsThy people shall be mine; And we will kneel to praise one God Before one common shrine.

Our souls—ah! what shall part our souls?

In ties of love entwined, They will defy the spells and chains That even death can bind.

\section{PINK, CARNATION. \\ (Woman's Love.)}

Mav's love lives but with hope; while woman's heart Still echoes to the music of the past.

* * * * * *

A love all sacrifice and suffering; a star That gathers lustre from the gloom of night;

A martyr's fond idolatry; a faith

Baptized in tears, to sorrow consecrate.

\section{POL Y A N THUS. \\ (Pride of Riches.)}

I GRIEVE to see thee vain and proud-I grieve That this world's honours have enticed thy heart. 
Such haughty airs become thee not. For me,

I better love a modest mien and look

Than all the gaudy tinsel wealth can buy,

Or vanity display. Put by thy pride, And by a holy life earn nobler praise Than such as pomp and idle show can win.

\section{PRIMROSE, EVENING.*}

(Inconstancy.)

Hast forgotten the days, love, the long-vanished days,

When our spirits communed through the bird and the flower;

When the stars linked our tlioughts by their glittering rays,

In a chain that lad more than electrical power?

Those days were the violet blossoms of love-

Young flowers that have faded and shrunk from thy view;

But though withered, forgotten, to thee they may prove,

They are pressed to one heart ever faithful and true.

* This plant is from one to two feet high. Flowers of various colours, and open suddenly. 


\section{ROSE, BRIDAL.* \\ (Happy Love.)}

IT has been said that love doth bind the heart

More strongly to the fading things of earth.

Not so with us : our spirits have no part

With feelings which are but of mortal birth. We love for heaven-let heaven become our home, Ere yet the angel beckon us to come.

And are you happy? asks some gentle one

In low, soft accents, and with thoughtful eye. Yes, dear; and more than happy, though the sun

Is softly clouded, and the deep blue sky

Grows deeper that it is not flushed with light,Though all the clouds that shade it are of white.

\section{ROSEBUD, MOSS.†}

(Confession of Love.)

I no believe that unto thee Trutl, honour, plain sincerity, Are jewels far before

* This belongs to the Bramble family. The flowers are small, white, double, and very beautiful.

t The Moss Rosebud is distinguished for its beauty. 
All that the others think are dear; And yet far more than they, I fear, Because I love thee more!

And yet I hope, beeause I love With thoughts that set thee far above

Vain Fortune's glittering store; Others may deem thou eanst be won By things that sparkle in the sun, But oh! I love thee more!

\section{ROSE, DEEP RED.}

(Bashful Love.)

The blushing rose that hangs its head, Or meets the sun with shrinking dread, Conceals within its heart a flame Whieh from that glowing noontide eame.

So have $I$ loved; but some strauge spell Forbids my heart its tale to tell: Here,--take this simple rose, and feel The love my lips dare not reveal.

\section{ROSE, FULL RED.}

(Beauty.)

Тну looks how lovely! and thy faee So eloquent with mental graee! 
Thy motions are as light and free As zephyrs o'er a summer sea. Thou art, in truth, a wayward child,Thy words so gay, thy steps so wild; And none call see thee speak or nnove Without some glow akin to love.

\section{SUNFLOWER, DWARF.}

(Adoration.)

Tine rose needs not the summer light,

The bird needs not the sheltering tree, So much as I, in sorrow's night,

Need smiles from thee.

Oh! never let thine eye grow cold,

Thy cherished voice grow rude to me;

But let thy lip, as oft of old,

Still smile on me.

\section{SWEETWILLIAM.}

(Gallantry.)

THe knights of old might envy thee Thy courtly grace of mien; Thy noble daring, brave and free, In every dangerous scene. 
To age how kind thy courtesy,

To woman how sincere!

Alike removed from vanity,

From artifice and fear.

\section{S Y R I N G A.}

(Memory.)

O Memory! thou ouly wakener of the dead !

Thou only treasurer of the vanished past!

How welcome art thou when bright hope is fled,

And sorrow's mantle o'er the soul is cast!

Back o'er those days, too beautiful to last,

Thy gentle hand will lead the saddened thought;

And though tlie tears may trickle warm and fast,

Yet thy sweet pictures with such peace are fraught,

The leart, beguiled, exclaims_." This is the fount I sought!"

\section{TULIP, VARIEGATED.* \\ (Beautiful Eyes.)}

Melting, dazzling, tender, bright,

Full of Love's own gentle light;

* Corolla bell-shaped. No calyx. Colour of the flower, in its natural state, crimson. By cultivation it has been made to assume every varioty of hue. 
Now downcast, and now uplifted,

With a world of beauty gifted;

Drooping now with silent thought,

Now with joy and gladness fraught;

Arch and mirthful, soft and pensive,

Now assailing, now defensive,- -

Filled with glory from the skies-

Ah! who can describe thine eyes ?

\section{TULIP, YELLOW.}

(Hopeless Love.)

I CANnot reproach thee! A malison rest

On the word that would wound thosc kind feelings of thine!

Wert thou colder than snow on Mouadanock's crest, The star of my love on thy spirit should shine.

Yet thou lovest me not as thou lov'dst me of yore;

A cloud lath ariscn and passed o'cr the light.

It is wcll! May God bless thee, dear friend, cvermorc;

So the sun but gild thee, I can wclcome the night.

\section{VERBENA.}

(Sensitiveness.)

Thine eye at others' sorrow weeps, Thy lip at others' joy looks gay; 
Thy heart's deep fount of feeling keeps In gentle, yet perpetual play.

The charms of nature thrill thy soul,

For nature's own true child thou art;

And waves of earnest feeling roll

In ceaseless music through thy heart.

\section{VERONICA.}

(Fidelity.)

There are some spirits fitly strung

To echo back the tones of mine;

And those few cherished souls among,

I dare, dear friend, to number thine.

Angels attend thee! May their wings

Fan every shadow from thy brow;

For only bright and loving things

Should wait on one so good as thou.

\section{VIOLET, BLUE.}

(Faithfulness.)

Ori, shame may come upen thy name, And want and suffering dim thine eye; 
Eut thou wilt find me still the same-For love like mine can never die.

I will be thine through weal and woe, Through days of joy and sorrow's night;

My faith like morning's beams shall glow,My love shall be thy quenchless light.

\section{VIOLET, PURPLE.}

(You occupy my Thoughts.)

Tпе smn is bright-its golden rays

Gild mountain-top and flower;

O'er rock, and ware, and vale it plays,

From morn till evening hour.

But, ah! no beauty in its beams

My weary heart can see,

While rocks, and vales, and glancing streams

Keep me away from thee!

The waves to others wear a light

More glorious than the sky;

To me earth's hues are only bright

Reflected from thine eye.

The world may deem me dull and sad-

I care not how that be; 
I never can nor will be glat, My love, away from thee!

\section{WA L L F LOWER.*}

(Fidelity in Misfortune.)

Av emblem true thou art

Of love's enduring lustre, given

To chcer a lonely heart.

Flower of the solitary place!

Gray Ruin's golden crown,

That lendest melancholy grace

To haunts of old renown,

'Thou mantlest o'er the battlement

By strife or storm decayed;

And fillest up each cnvious rent

'Time's canker tooth hath made.

\section{WOODRUFF. \\ (Modest Worth.)}

THINe excellence is of a rare degree;

Though praised by others, 'tis unknown to thec.

* This is a beautiful, fragrant flower, growing ujon old walls, and among the ruins of ciastles and abbeys. 
In humble deeds of love and kindly care

To those who in carth's riches own no share;

By acts of mercy all unseen of men;

By silent victory over pride and sin;

by faith, and lope, and charity on earth,

Thou prov'st to others thy transccudent worth;

Wliilst to thyself thy goodness is unknown-

Though virtue crowns and claims thee for her own.

\section{HYMN TO THE FLOWERS.}

DAY STARS! that ope your eyes with morn to twinkle From rainbow galaxies of Earth's creation, And dew-drops on her lovely altars sprinkle As a libation!

Ye matin worshippers! who, bending lowly

Before the uprisen sun, God's lidless eye, Throw from your chalices a swcet and holy Incense on lighl !

Ye bright mosaics! that with storied beauty 'The floor of Nature's temple tesselate, What numerous emblens of instructive duty Your forms create! 
'Neath cloistered boughs each floral bell that swingeth,

And tolls its perfume on the passing air, Makes Sabbath in the fields, and ever ringeth

\section{A call to prayer.}

Not to the domes, where crumbling arch and column Attest the feebleness of mortal hand;

But to tliat fane, most catholic and solemn, Which God hath plawned;

To that catliedral, boundless as our wonder,

Whose quenchless lamps the sun and moon supplyIts choir the wind and waves, its organ thunder, Its dome tlie sky:

There, as in solitude and shade I wander Through the green aisles; or stretched upon the sod; Awed by the silence, reverently ponder The ways of God,-

Your voiceless lips, O flowers! are living preacher's,

Each cup a pulpit, and each lcaf a book, Supplying to my fancy numerous teachers, From loneliest nook.

Floral apostles! that, in dewy splendour, "Weep without woe, and blush without a crime," 
Ol, may I deeply learn, and ne'er surrender, Your lore sublime!

"Thou wert not, Solomon, in all thy glory, Arrayed," the lilies cry, "in robes like ours! How vain your grandeur! ah, low transitory Are human flowers!"

In the sweet-scented picture, lieavenly Artist, With which thou paintest Nature's wide-spread hall, What a delightful lesson thou impartest Of love to all!

Not useless are ye, flower's! though made for pleasurc,

Blooming o'cr field aud wave by day and night; From every source your sanction bids me treasure Harmless delight.

Ephemeral sages! what instructors hoary

For such a world of thought could furuish scope! Each fading calyx a memento mori, Yet fount of hope.

Postliumous glories! angel-like collection!

Upraised from seed or bulb interred in earth, Ye are to me a type of resurrection,

A sccond birth! 


\section{Were I, O God, in churchless lands remaining, Far from all voice of teachers or divines, $\mathrm{My}$ soul would find in flowers of thy ordaining, Priests, sermons, shrines:}

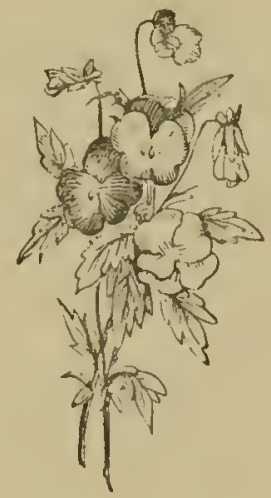




$$
18
$$


CURATORIIL REV. 11197 - NO ACTION 


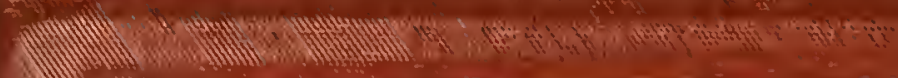

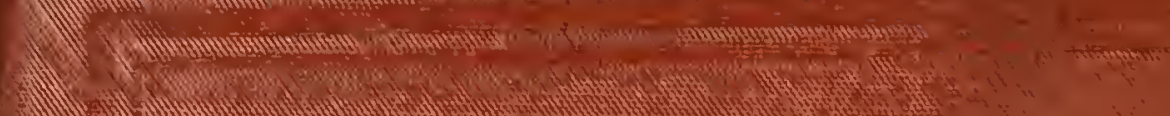
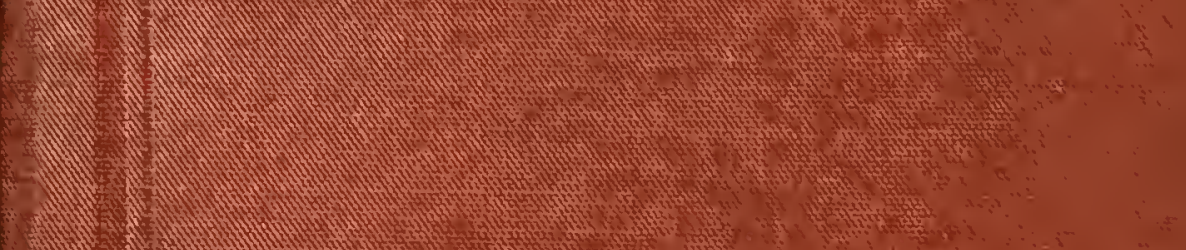

int.

H.

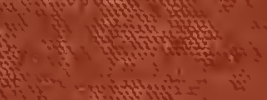

(n)

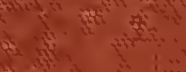

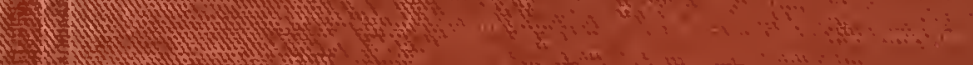

How

HW H H

(i.

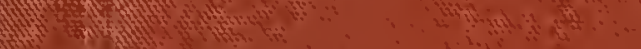

iniv

int.

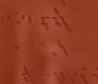

$x^{2}$ 